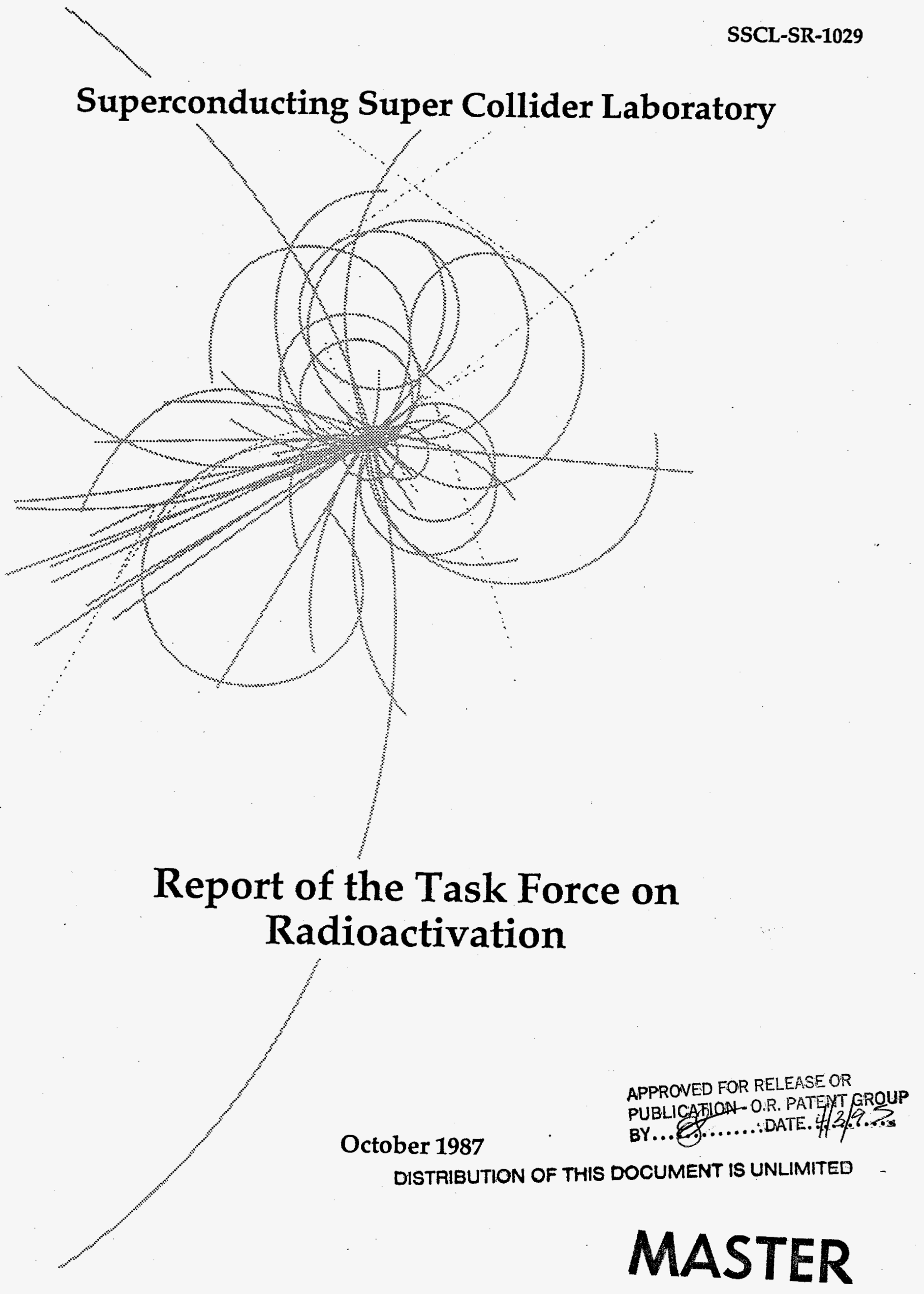




\title{
REPORT OF THE TASK FORCE ON RADIOACTIVATION
}

\author{
October 1987
}

\section{DISCLAIMER}

This report was prepared as an account of work sponsored by an agency of the United States Government. Neither the United States Government nor any agency thereof, nor any of their employees, makes any warranty, express or implied, or assumes any legal liability or responsibility for the accuracy, completeness, or usefulness of any information, apparatus, product, or process disclosed, or represents that its use would not infringe privately owned rights. Reference herein to any specific commercial product, process, or service by trade name, trademark, manufacturer, or otherwise does not necessarily constitute or imply its endorsement, recommendation, or favoring by the United States Government or any agency thereof. The views and opinions of authors expressed herein do not necessarily state or reflect those of the United States Government or any agency thereof.

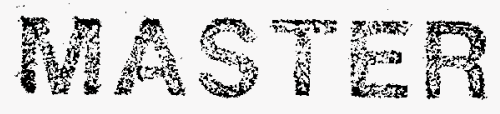




\section{DISCLAIMER}

Portions of this document may be illegible in electronic image products. Images are produced from the best available original document. 


\section{TABLE OF CONTENTS}

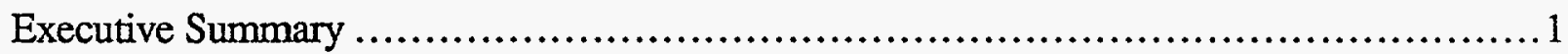

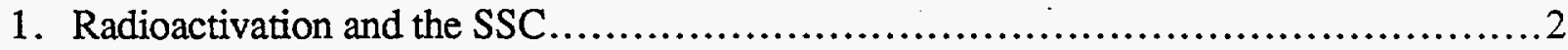

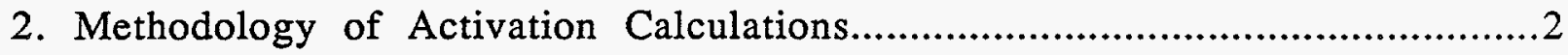

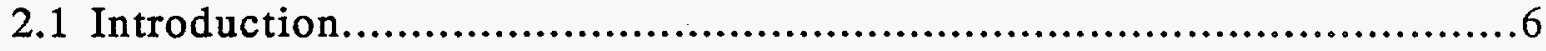

2.2 Calculation Procedure ......................................................... 10

2.3 Final-State Isotopes of Interest............................................... 14

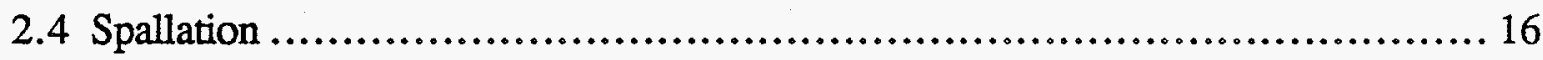

2.5 Approximate Methods....................................................... 16

2.6 Input for Example Calculations ................................................. 17

2.7 A Worked Example: Activity in the Ring Magnets................................. 18

2.8 Absorbed Dose ..................................................................... 19

2.9 A Worked Example: Absorbed Dose at the Surface of an SSC Dipole .............. 23

3. Abort Beam Dumps ........................................................ 25

3.1 Activaton Levels in the Abort Dumps ....................................... 25

3.2 Handling and Dismantling of the Abort Beam Dumps ............................. 29

3.3 Disposal of Radioactive Water from the Abort Dump Cooling System ............. 31

4. Activation in the Injection System............................................... 32

5. Beam Losses in the Storage Rings and at the Interaction Regions .................... 36

5.1 Superconducting Magnets in the Storage Rings.......................................... 37

5.2 Beam Collimators and Scrapers.................................................... 38

5.3 Interaction Regions - Experimental Apparatus................................. 42

6. Comparison to Previous Decommissioning Experience ........................... 48

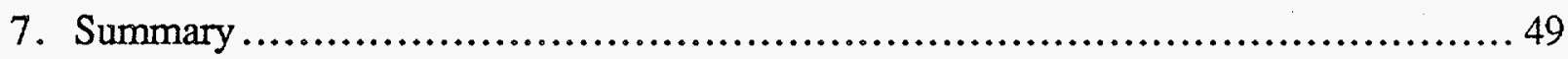

8. Acknowledgements .............................................................. 49

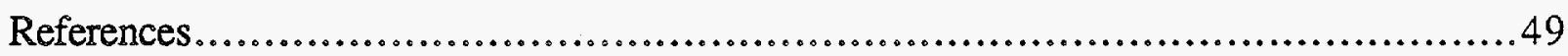

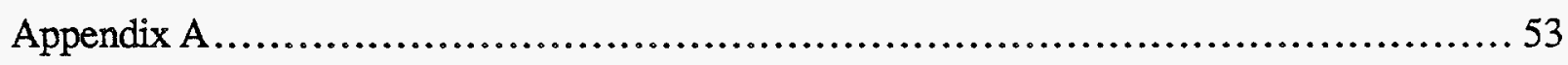

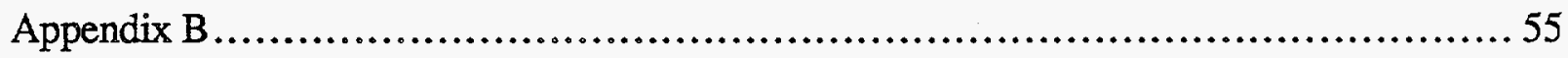

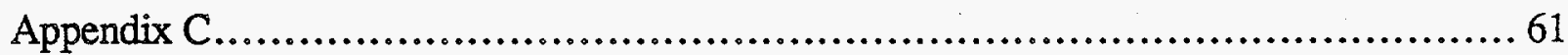

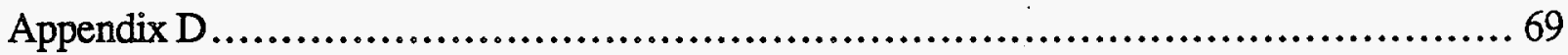

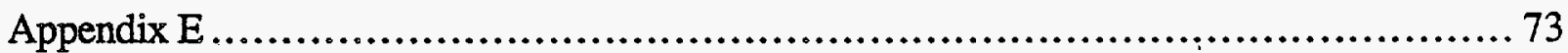

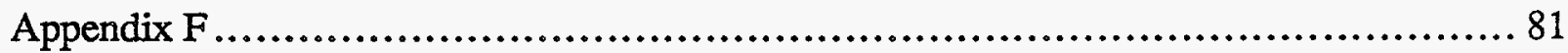

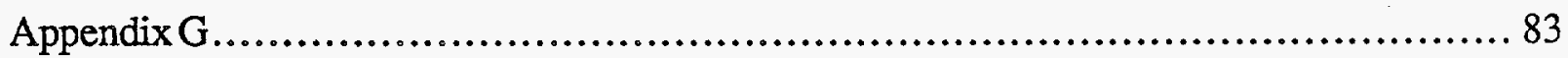




\section{EXECUTIVE SUMMARY}

Estimates have been made of the residual radioactivation of the components of the SSC accelerator and detector complex. Such activation is caused by the loss of a small fraction of the proton beams being transported in the SSC accelerator system, by deliberate removal (dumping) of the beams and by particles created in the proton-proton collisions in the SSC storage rings.

Existing methodology that has been successfully used to determine activation levels at other accelerator facilities has also been used to estimate the activation of SSC components in those cases where calculational methods may be applied. In addition, we have used measurements from existing accelerators, primarily the Fermilab and CERN accelerators, to provide the means to estimate activation for those cases in which calculational methods, such as Monte Carlo shower codes, are difficult to apply. We have also used these measurements to check the calculations from the shower codes where possible.

We have estimated activation levels and dose rates, where possible, for the following components of the SSC: the abort dumps for both the storage rings and the elements of the injector system; beam collimators and scrapers; magnets and other apparatus in the region of injection/extraction from one accelerator to another, target stations used to create test beams from the high energy booster, superconducting magnets in the high energy booster and the storage rings; and representative detector elements.

We also briefly discuss aspects of handling or dismantling the components most significantly activated and the relevance to decommissioning of the SSC complex. A short description of the decommissioning of the Intersecting Storage Rings at CERN, the only pp collider that has been decommissioned, is included in this report.

Radioactivation of SSC components will be qualitatively and quantitatively similar to activation at existing large accelerator facilities for high energy physics such as Fermilab or CERN. The higher energy of the SSC will not present new problems in the routine monitoring and handling of activated components during either the operational phase of the SSC or during a possible decommissioning process. 


\section{RADIOACTIVATION AND THE SSC}

The purpose of this Task Force was to describe those components of the Superconducting Super Collider (SSC) complex which might become significantly radioactive during the life of the SSC and to describe briefly the associated radiation levels and the handling and disposition of these components. The Task Force was charged to estimate the appropriate radiation levels using new calculations and experience from existing or previous accelerators as necessary. A list of the members of the Task Force is given in Appendix A.

The accelerator complex (see Fig. 1.1) for the SSC ${ }^{1}$ includes a Linac, a Low Energy Booster (LEB), a Medium Energy Booster (MEB), a High Energy Booster (HEB), and two storage rings providing pp collisions at an energy of $40 \mathrm{TeV}$. The Linac, LEB, and MEB are very similar to existing accelerators at Fermilab and Brookhaven in the United States, to machines at CERN in Europe, to accelerators at KEK in Japan, and to accelerators that are no longer operational and which have been dismantled. The HEB primarily contains superconducting magnets and is similar to the Tevatron at Fermilab, operating in a fixed target mode. The main storage rings are conceptually similar to the Tevatron operating in its storage ring mode but are substantially larger in size and will provide higher intensity interactions.

Previous studies have been made of the environmental radiation shielding for the SSC and include a general description of the sources of radiation. ${ }^{2}$ In this report we will discuss those few areas of the SSC complex that may have significant residual radioactivity after the SSC has shutdown. These are (see Fig. 1.1 for a guide to the location of some of these items):

(1) beam dumps associated with the injectors to the storage ring and the two abort beam dumps for the storage rings

(2) magnets and associated hardware and enclosures near the regions of beam transfer from one accelerator to another and in the vicinity of beam-abort regions

(3) beam scrapers in the storage rings, which are used to remove a small fraction of the stored beam during the operation of the storage rings and during and after injection from the HEB 

(4) targets for the production of high-energy test beams

(5) collimators located among the interaction region magnets which prevent quenching of the superconducting magnets in the interaction regions

(6) detector components located near the beam pipe in the interaction regions

The superconducting magnets in the HEB and in the storage rings will not become significantly radioactive since the amount of energy deposition required to induce significant radioactivity is much greater than the amount which would result in quenches of the magnets. This is discussed in more detail in Sec. 5.

In order to estimate the activation of the components, typical operating parameters of the SSC must be known. For our estimates we have chosen to be conservative and have followed Ref. 2 in assuming a stored beam intensity in the collider rings approximately three times greater than the present design value, which would yield a luminosity ten times the design value of $10^{33} \mathrm{~cm}^{-2} \mathrm{sec}^{-1}$. In particular we have assumed for our estimates:

- a maximum of $4 \times 10^{14}$ protons stored in each of the rings per fill

- a total of $2 \times 10^{17}$ protons per year accelerated to $20 \mathrm{TeV}$ in each collider ring

The intensity requirements in the injector systems follow immediately from these assumptions. Activation of injection/extraction components is difficult to model. Therefore, in order to estimate the effects due to injection/extraction losses, we have relied on experience at the Fermilab Tevatron extrapolated to the SSC.

In the subsequent sections we describe in detail our estimates of the residual radioactivity induced in the relevant components of the SSC complex. We also comment on the handling of these components resulting from servicing needs or from dismantling at the end of the useful life of the SSC. In the latter case, there has been substantial experience with previous accelerators, ${ }^{3}$ most recently with the Intersecting Storage Ring (ISR) at CERN which was also a pp collider. The experience of decommissioning the ISR is reported in Sec. 6, and in Appendices 
$\mathrm{D}$ and $\mathrm{E}$ and references therein. Decommissioning of the SSC complex will not be qualitatively different from previous experience.

When discussing components of the SSC complex such as concrete in the tunnel walls or concrete or soil surrounding the various dumps, it is important to recognize that naturally occurring radioactivity will already be present in the soil and building materials. Although there are variations in these natural levels, representative values for soil are given in Table 1.1 and for some building materials in Table 1.2 below. 4

TABLE 1.1 Approximate Levels of Naturally Occurring Radioactivity in Soil

NUCLIDE

$40 \mathrm{~K}$

${ }^{232} \mathrm{Th}$

$238 \mathrm{U}$
CONCENTRATION (pCi/gm)

12

1

0.6

Both ${ }^{232} \mathrm{Th}$ and ${ }^{238} \mathrm{U}$ are largely in equilibrium with their decay products and hence represent a total radioactivity about ten times larger than that shown in the Table

TABLE 1.2 Radioactivity in Some Building Materials in pCi/gm

\begin{tabular}{llcl}
\hline MATERIAL & $40 \mathrm{~K}$ & $226 \mathrm{Ra}$ & $232 \mathrm{Th}$ \\
\hline Bricks & 16 & 1.5 & 2.2 \\
Concrete block & 5.4 & 0.4 & 1.1 \\
Gypsum & - & - & 0.3 \\
Rock wool & 3.8 & - & - \\
\hline \hline
\end{tabular}




\section{METHODOLOGY OF ACTIVATION CALCULATIONS}

\subsection{Introduction}

Thomas and Stevenson have given a succinct description of the production of radioactivity at particle accelerators: ${ }^{5}$

"In the interaction of a hadron of sufficient energy with a nucleus an intranuclear cascade is generated. The emission of particles produced during this cascade from the nucleus (spallation) is followed by the evaporation of nucleons from the excited residual nucleus. The number of radionuclides that might be produced is in principle very large, but in practice only a relatively small number are of consequence in radiological protection. Although, in principle, all the nuclides that have a mass number and atomic number less than or equal to the sum of these numbers for the projectile and target nuclei may be generated, in practice it is found that the number of radionuclides of concern in radiological protection is limited by a combination of production cross section and radioactive half life."

While the general problem involves concerns such as personnel protection during maintenance and modification, the focus of this report is more circumscribed. The interval during which machine components are exposed to radiation from the beams is long, perhaps several decades. Although the beam intensity is unlikely to be constant, we make the conservative assumption that there is a constant proton loss rate at the highest beam intensities presently envisaged. Most isotopes of interest (including tritium) have lifetimes sufficiently short that equilibrium concentrations will have been reached under these assumptions.

At the moment of turnoff, activities with half-lives ranging from less than a second to many years are present in important quantities. A few minutes later, only isotopes with lifetimes in excess of a minute or so exist in measurable 
concentrations. * Although the details depend upon the irradiated material, the general picture is that the total activity decreases very quickly at first, then progressively more slowly as longer-lived activities come to dominate. As an example, the contribution of various radionuclides to the surface dose of a cylindrical iron beam stop irradiated by an on-axis $200 \mathrm{GeV}$ proton beam is shown in Fig. 2.1. ${ }^{6}$ The "initial level" is defined (here and elsewhere) as the level $6 \mathrm{~min}(0.1 \mathrm{~h})$ after turnoff, when very short-lived activities have already reached negligible concentrations. ${ }^{\dagger}$ As can be seen, the activity after 4 days is about half of the "initial" level. After a month most of the ${ }^{52} \mathrm{Mn}$ (5.6 day half-life) and ${ }^{48} \mathrm{~V}$ (16 days) have also decayed, leaving mostly ${ }^{54} \mathrm{Mn}$ activity at about $\frac{1}{4}$ the initial total level.

Similar situations occur for other materials. We conclude that there is a great advantage in delaying access for decommissioning purposes by a few days or few weeks, particularly to high-dose areas. On the other hand, the ubiquitous presence of longer-lived radionuclides ( $Z 1 \mathrm{yr}$ ) makes further delay only marginally advantageous. We therefore assume this scenario, and assign prime importance to those activities which have appreciable concentrations after 10 days or so.

To illustrate the calculation methodology developed below, it is useful to pursue the case of ${ }^{54} \mathrm{Mn}$ induced in iron one step further. We consider only the ${ }^{54} \mathrm{Mn}$ activity induced in iron by a long, uniform exposure, and we assume knowledge of the spectra of low-energy particles (thermal energies to a few hundred $\mathrm{MeV}$ ) produced in the nuclear cascades. For incident neutrons with energies between $500 \mathrm{MeV}$ and $730 \mathrm{MeV}$, the average cross section for producing ${ }^{54} \mathrm{Mn}$ in iron is about $30 \mathrm{mb} .{ }^{7} \ddagger$ Given a cross section $\sigma_{\text {inel }} \approx 700 \mathrm{mb}$ for

* For example, we might expect ${ }^{54} \mathrm{~V}$ in irradiated iron. Its half-life is $49.8 \mathrm{~s}$. After 10 minutes, its concentration has dropped to $0.024 \%$ of that present at turnoff. After 20 minutes, only 0.056 parts per million of the original activity remains.

$\dagger$ In some cases, the decay products of short-lived species are themselves important longlived activities. For example, ${ }^{41} \mathrm{Ca}$ is the decay product of the $0.87 \mathrm{~s}$ nuclide ${ }^{41} \mathrm{~S}$.

\$ Mainly through the ${ }^{54} \mathrm{Fe}(\mathrm{n}, \mathrm{p}){ }^{54} \mathrm{Mn}$ and ${ }^{56} \mathrm{Fe}(\mathrm{n} ; 2 \mathrm{np}){ }^{54} \mathrm{Mn}$ reactions. 


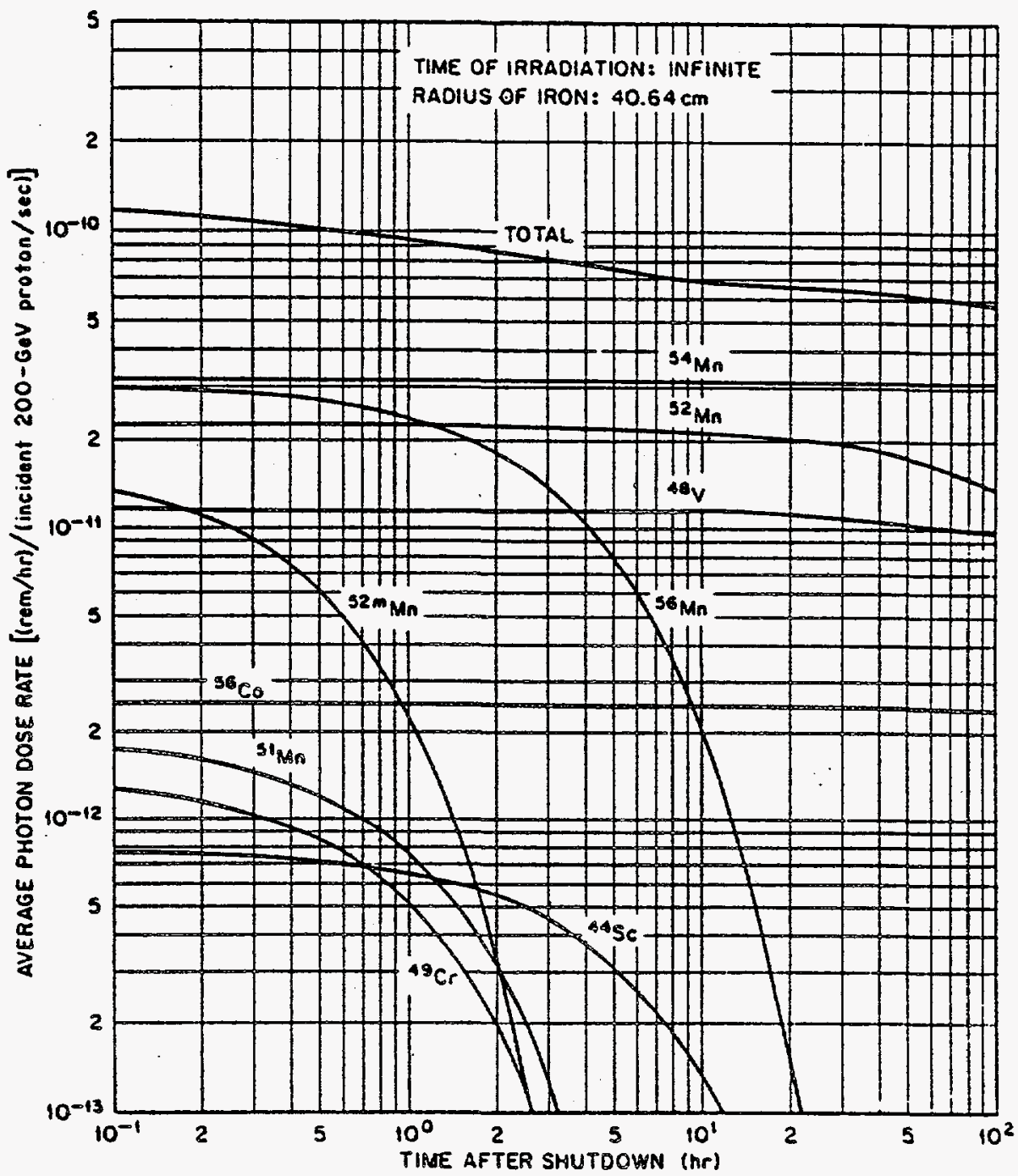

Figure 2.1. Contribution of various radionuclides to the average total photon dose rate at the surface of an iron beam stop. $200 \mathrm{GeV}$ protons are incident on the axis, and the radius is $40.64 \mathrm{~cm}$. Reproduced from Fig. 8 of Ref. 6 .

inelastic iron-neutron scattering, it follows that about $4.3 \%$ of the iron-nucleus collisions will result in ${ }^{54} \mathrm{Mn}$ production. Now suppose that $R d t^{\prime}$ atoms of ${ }^{54} \mathrm{Mn}$ are produced in time interval $d t^{\prime}$ at a time $t^{\prime}$ in the past. "Past" is with respect to some reference time, e.g. accelerator shutdown. At a time $t$ in the future,

$$
N\left(t, t^{\prime}\right)=R d t^{\prime} \mathrm{e}^{-t^{\prime} / \tau} \mathrm{e}^{-t / \tau}
$$

atoms remain, where $\tau$, the decay time, is given by the half-life divided by $\ln 2$. 
(The half-life of ${ }^{54} \mathrm{Mn}$ is $0.797 \mathrm{yr}$, so $\tau=1.15 \mathrm{yr}$.) If the production rate $R$ were constant for a total time $T$ in the past, then

$$
N(t)=\tau R\left(1-\mathrm{e}^{-T / \tau}\right) \mathrm{e}^{-t / \tau}
$$

atoms remain at time $t$. In many case of practical interest (including the present example), $T>>$, and $\left(1-\mathrm{e}^{-T / \tau}\right) \approx 1$; the total "on time" is immaterial. Equilibrium has been reached under these conditions and the production rate $R$ is equal to the decay rate. ${ }^{\star} t$ The production rate must be integrated over incident hadron energies to obtain the final result. Surprisingly, low energies dominate. In a $20 \mathrm{TeV}$ proton collision with iron, less than $3 \%$ of the interactions with iron nuclei are initiated by particles with energies greater than $3 \mathrm{GeV}$.

${ }^{54} \mathrm{Mn}$ emits a $0.83 \mathrm{MeV} \gamma$-ray in the course of decaying to the stable isotope ${ }^{54} \mathrm{Cr}$. If the distribution of ${ }^{54} \mathrm{Mn}$ atoms in the iron is known, one may calculate the $\gamma$ flux and dose at a given point in or on the target by taking into account $\gamma$ absorption and scattering.

This example contains most of the elements of a "real" calculation of radioactivation and dose. Its generalization is complicated by the wealth of possible reactions, the lack of complete cross section measurements, and the lack of knowledge of the incident hadron spectra. Even here, the cross section reported at $340 \mathrm{MeV}$ is not really consistent with those measured at higher energies, ${ }^{7}$ and low energy neutrons are responsible for more than half of the ${ }^{54} \mathrm{Mn}$ production. ${ }^{6}$

* The identity is particularly simple if the activity is expressed in becquerels, or disintegrations per second. As is done elsewhere in this report, it is often convenient to convert to curies, where $1 \mathrm{Ci}=3.7 \times 10^{10} \mathrm{~Bq}$.

$\dagger$ At the other extreme where $\tau \gg T$, the activity is $(T / \tau) R$. For example, ${ }^{7} \mathrm{Be}\left(t_{1 / 2}=\right.$ $53 \mathrm{~d})$ and ${ }^{10} \mathrm{Be}\left(t_{1 / 2}=1.6 \times 10^{6} \mathrm{y}\right)$ are produced in almost equal numbers by cascades in a carbon target. If the activity of ${ }^{7} \mathrm{Be}$ is $R$, then that of ${ }^{10} \mathrm{Be}$ after a 25 year exposure is $8 \times 10^{-6} R$. It is for this reason that the production of very long-lived activities can usually be neglected. 
In spite of such practical complications, the activation level expected for various SSC components may confidently be calculated to the desired level of accuracy. The keyword is "desired level of accuracy:" a factor of about two may be obtained. This uncertainty is likely to be smaller than the uncertainty in exposure estimates for collimators, septa, dipoles, etc. over the lifetime of the machine.

\subsection{Calculation Procedure}

Computational methods are based upon a large number of calculations for other accelerators and comparison with measurements. ${ }^{5}$ They are best applied in situations with relatively simple geometry and well known losses. In the SSC we have applied these methods to the abort beam dumps for the storage rings, various collimators/scrapers, and experimental apparatus. In those cases with complicated geometry and/or losses which depend in detail upon fine-tuning the operation of the machine, we have relied on experience and measurements at the Fermilab Tevatron (See Section 4).

Given an object (e.g. a magnet) where predictable beam loss occurs, the full procedure for calculating residual activation might be as follows:

1. Calculate the hadronic cascade(s) using any one of several programs developed for the purpose (CASIM, ${ }^{8}$ FLUKA, ${ }^{9}$ HETC,${ }^{10}$ MARS, ${ }^{11}$ etc.). The output is "star density," the density of inelastic collisions in the material with incident hadronic energies above some cutoff, usually about $50 \mathrm{MeV}$. The total number of stars per incident hadron is usually about 2 stars per $\mathrm{GeV}$ at several hundred $\mathrm{GeV}$. The number of stars scales with energy roughly as $E^{0.8} .^{5}$

Either directly or with minor modifications, these programs also yield hadronic energy spectra down to the cutoff energy.

When a particle's energy drops below the cutoff, it is either tallied or its vital statistics written to an output file for further analysis. 
A brief discussion of these codes and a comparison of simulation and experimental results is given in Ref. 2 .

2. The output file is processed by a low-energy transport program such as MORSE. ${ }^{12}$ Such programs are designed to handle the complexities of nuclear energy levels, and they access one of several extensive data libraries such as VITAMIN-E. ${ }^{13}$ A major simplification at low energies is that only neutrons need to be transported, since in this energy range charged particles quickly stop.

$2^{\prime}$. An alternative to detailed low-energy propagation is available, although some approximation is involved: It has long been observed that under suitable conditions (far from the cascade initiation point and the cascade axis, and far from material boundaries), the spectrum is "universal:" The hadron composition and the spectrum of each hadronic variety is independent of incident hadron type and, except for an overall scale factor, independent of position and incident hadron energy. ${ }^{14-18}$ The usefulness of this "universal spectrum" follows from our earlier observation that the vast majority of radionuclides are produced in low-energy collisions, so that there is little sensitivity to details of the high-energy spectra. An example of this composition and the spectra of the components in soil is shown in Fig. 2.2. ${ }^{14}$ To the extent that the approximation is true, the low-energy transport calculation need be done only once per material . The scale factor is set by a measure of the cascade intensity, such as star density.

3. Given the spectrum and composition, the procedure outlined at the beginning of this section is then used to calculate the activation level. Unfortunately, the production cross section is for an incident hadron variety $h$, a target nucleus of type $i$, and a daughter species of type $f$. Moreover, it depends upon the energy $E$ of the hadron incident on the target nucleus. In obvious notation, we label the cross section $\sigma_{h i f}(E)$. 


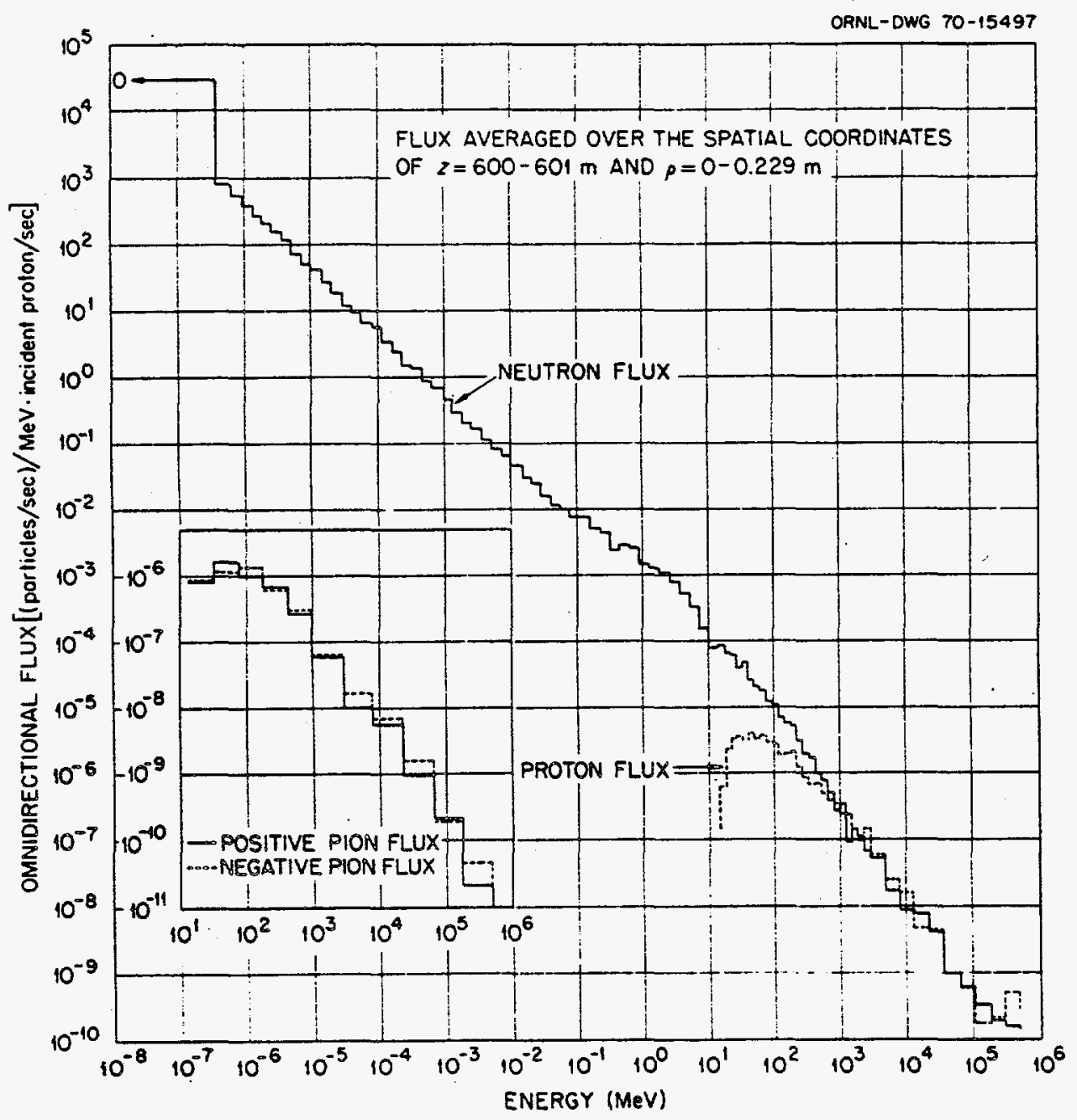

Figure 2.2. Kinetic energy spectrum of hadrons as calculated by T. A. Gabriel and $R$. T. Santoro for the soil backwall of a target-tunnel configuration. ${ }^{14}$ This spectrum was assumed to be a representative "universal" spectrum by Van Ginneken and Awschalom in calculating the effects of low energy particles not followed by CASIM. ${ }^{15}$

Let the flux of hadrons of kind $h$ be $\phi_{h}(E)$. If $\mathcal{N}_{i}$ is the number density of the $i$ th nuclear species present in the target, then the total production rate $R_{i f}$ for the $f$ th final state species is

$$
R_{i f}=\sum_{h} \int_{E} \mathcal{N}_{i} \sigma_{h i f}(E) \phi_{h}(E) d E
$$

If more than one target isotope can produce the final isotope, then the summation should also be carried out over $i$. The result is $R_{f}$, the production rate per unit volume of species $f$ in a given material. In 
the usual case, only one or a few target nuclides contribute appreciably to $R_{f}$.

Similarly, if $\sigma_{h i \text { inel }}(E)$ is the inelastic cross section for hadron variety $h$ on target species $i$ at energy $E$, then the rate of star production per unit volume by target hadrons with energy in excess of $E_{\min }$ is

$$
S=\sum_{i} \sum_{h} \int_{E_{\min }} \mathcal{N}_{i} \sigma_{h i \text { inel }}(E) \phi_{h}(E) d E
$$

The ratio

$$
r_{f}=R_{f} / S
$$

is the fraction of stars in which nuclide $f$ is produced. An implicit subscript labeling the material exists, since the result is different for natural iron, concrete, etc.

Again following the development of the introduction, the number density of atoms of isotope $f$ present at a time $t$ after the end of steady-state operation is given by

$$
\begin{aligned}
N_{f}(t) & =\tau_{f} R_{f} \mathrm{e}^{-t / \tau_{f}} \\
& =S r_{f} \tau_{f} \mathrm{e}^{-t / \tau_{f}}
\end{aligned}
$$

where $\tau_{f}$ is the decay time (half-life/(ln 2)) and it is assumed that the accelerator operated for a time $T>\tau_{f}$. The number of disintegrations per unit time in a unit volume is $N_{f} / \tau_{f}$. At $t=0$ it is $R_{f}$; this is just a re-statement of the fact that in equilibrium the decay rate equals the production rate. The specific activity, or number of disintegrations per unit time per unit mass, may be obtained by dividing by the density of the material. Obviously, the appropriate sum over the final radioisotopes of interest must be carried out to obtain the final specific activity. 
Although the universal spectrum situation discussed in item $2^{\prime}$ above has not been assumed so far, it is evident that this approximation is equivalent to the factors $r_{f}$ being "universal." They might be tabulated (once and for all, pending new experimental data and upgraded transport programs) for the commonest target materials $(\mathrm{C}, \mathrm{Fe}, \mathrm{Cu}, \mathrm{Pb}$, concrete, ...) and the most interesting radioactive daughters $\left({ }^{3} \mathrm{H},{ }^{54} \mathrm{Mn},{ }^{60} \mathrm{Co}, \ldots\right)$. Activation levels could then be obtained in some detail from star density alone.

The total activity can be obtained by integrating $N_{f}(t)$ over the volume of interest. To the extent that the universal spectrum approximation is valid, it is proportional to the total star density $\int S d^{3} x$.

Several straightforward but tedious complications have been ignored in this treatment, notably the occasional production of an important longlived isotope from the decay of a collision product. Such details are discussed in the literature. ${ }^{6}$

\subsection{Final-State Isotopes of Interest}

Fortunately, only a small number of low- $Z$ radionuclei exist whose lifetimes are long enough to be of concern. Ref. 19 provides a table of all isotopes with $Z$ less than 28 which have half-lives longer than one day; this table is reproduced here as Table 2.1. Only 15 of the isotopes have half-lives longer than a day, and only a few of these are of concern. (The table was presumably truncated at $Z=27$ because of the large number of long-lived nickel activities. ${ }^{60} \mathrm{Co}$ should be added; its half-life is 5.272 years.)

Several heavier elements are of interest in this study. Some stainless steels contain substantial quantities of nickel and (infrequently) cobalt, and copper is also relatively common in the accelerator environment. Irradiation of these elements produces significant amounts of ${ }^{60} \mathrm{Co}$ by means of several reactions, e.g. ${ }^{59} \mathrm{Co}(\mathrm{n}, \gamma){ }^{60} \mathrm{Co}$. Important lead and thallium activities are induced by 
Table 2.1.

Radioactive nuclei $(Z<28)$ with half-lives $>1$ day (as reproduced from Table 2 of Ref. 19).

\begin{tabular}{|c|c|c|}
\hline Element & $\begin{array}{c}\text { Half-life } \\
\text { (years) }\end{array}$ & \\
\hline${ }^{3} \mathbf{H}$ & $1.23 \times 10^{1}$ & \\
\hline${ }^{7} \mathrm{Be}$ & $1.47 \times 10^{-1}$ & \\
\hline${ }^{10} \mathrm{Be}$ & $2.70 \times 10^{6}$ & \\
\hline${ }^{14} \mathrm{C}$ & $5.77 \times 10^{3}$ & \\
\hline $22 \mathrm{Na}$ & $2.58 \times 10^{0}$ & \\
\hline${ }^{* 26} \mathrm{Al}$ & $7.40 \times 10^{5}$ & $\left({ }^{26} \mathrm{Si} \stackrel{2 \sec }{\longrightarrow}{ }^{26} \mathrm{Al}+\beta^{+}\right)$ \\
\hline 32Si & $7.00 \times 10^{2}$ & ${ }^{32} \mathrm{Si} \rightarrow{ }^{32} \mathrm{P}+\beta^{-}$ \\
\hline${ }^{32} \mathrm{P}$ & $\left.3.92 \times 10^{-2}\right\}$ & $-\mathrm{SI} \rightarrow-\mathrm{p}+p$ \\
\hline 33p & $6.85 \times 10^{-2}$ & \\
\hline${ }^{35} \mathbf{S}$ & $2.37 \times 10^{-1}$ & \\
\hline${ }^{36} \mathrm{Cl}$ & $3.00 \times 10^{5}$ & \\
\hline${ }^{37} \mathrm{Ar}$ & $9.39 \times 10^{-2}$ & $\left({ }^{37} \mathrm{~K} \stackrel{1.2 \mathrm{sec}}{\longrightarrow}{ }^{37} \mathrm{Ar}+\beta^{+}\right)$ \\
\hline${ }^{39} \mathrm{Ar}$ & $2.60 \times 10^{2}$ & $\left({ }^{39} \mathrm{Cl} \stackrel{55.5 \mathrm{~min}}{\longrightarrow}{ }^{39} \mathrm{Ar}+\beta^{-}\right)$ \\
\hline${ }^{42} \mathrm{Ar}$ & $3.30 \times 10^{1}$ & $\left({ }^{42} \mathrm{Ar} \rightarrow{ }^{42} \mathrm{~K}+\beta^{-} \stackrel{12.4 \mathrm{~h}}{\longrightarrow}{ }^{42} \mathrm{Ca}+2 \beta^{-}\right)$ \\
\hline${ }^{40} \mathrm{~K}$ & $1.30 \times 10^{9}$ & \\
\hline${ }^{41} \mathrm{Ca}$ & $1.10 \times 10^{5}$ & $\left({ }^{41} \mathrm{~S} \stackrel{0.87 \mathrm{sec}}{\longrightarrow}{ }^{41} \mathrm{Ca}+\beta^{+}\right)$ \\
\hline${ }^{45} \mathrm{Ca}$ & $4.52 \times 10^{-1}$ & $\left({ }^{45} \mathrm{~K} \stackrel{20 \min }{\longrightarrow}{ }^{45} \mathrm{Ca}+\beta^{-}\right)$ \\
\hline${ }^{47} \mathrm{Ca}$ & $1.29 \times 10^{-2}-2$ & \\
\hline${ }^{* 44} \mathrm{Sc}$ & $6.57 \times 10^{-3}$ & ${ }^{47} \mathrm{Ca} \rightarrow{ }^{47} \mathrm{Sc}+\beta$ \\
\hline${ }^{* 46} \mathrm{Sc}$ & $2.30 \times 10^{-1}$ & \\
\hline${ }^{47} \mathrm{Sc}$ & $9.31 \times 10^{-3}$ & ${ }^{44} \mathrm{Ti}+\mathrm{e}^{--} \rightarrow{ }^{44} \mathrm{Sc}$ \\
\hline${ }^{48} \mathrm{Sc}$ & $5.02 \times 10^{-3}$ & \\
\hline${ }^{44} \mathrm{Ti}$ & $4.70 \times 10^{1}$ & \\
\hline${ }^{48} \mathrm{~V}$ & $4.41 \times 10^{-2}$ & $\left({ }^{48} \mathrm{Cr}+\mathrm{e}^{-} \stackrel{23 \mathrm{~h}}{\longrightarrow}{ }^{48} \mathrm{~V}\right)$ \\
\hline${ }^{49} \mathrm{~V}$ & $9.04 \times 10^{-1}$ & $\left({ }^{49} \mathrm{Cr} \stackrel{42 \min }{\longrightarrow}{ }^{49} \mathrm{~V}+\beta^{+}\right)$ \\
\hline${ }^{51} \mathrm{Cr}$ & $7.61 \times 10^{-2}$ & $\left({ }^{51} \mathrm{Mn} \stackrel{45 \mathrm{~min}}{\longrightarrow}{ }^{51} \mathrm{Cr}+\beta^{+}\right)$ \\
\hline${ }^{* 52} \mathrm{Mn}$ & $1.53 \times 10^{-2}$ & $\left({ }^{52} \mathrm{Fe} \stackrel{8 \mathrm{~h}}{\longrightarrow}{ }^{52} \mathrm{Mn}+\beta^{+}\right)$ \\
\hline${ }^{53} \mathrm{Mn}$ & $2.00 \times 10^{6}$ & $\left({ }^{53} \mathrm{Fe} \stackrel{9 \min }{\longrightarrow}{ }^{53} \mathrm{Mn}+\beta^{+}\right)$ \\
\hline${ }^{54} \mathrm{Mn}$ & $1.97 \times 10^{-1}$ & \\
\hline${ }^{55} \mathrm{Fe}$ & $2.70 \times 10^{\circ}$ & $\left({ }^{55} \mathrm{Co} \stackrel{18 \mathrm{~h}}{\longrightarrow}{ }^{55} \mathrm{Fe}+\beta^{+}\right)$ \\
\hline${ }^{59} \mathrm{Fe}$ & $1.23 \times 10^{-1}$ & \\
\hline${ }^{56} \mathrm{Co}$ & $2.19 \times 10^{-1}$ & \\
\hline${ }^{57} \mathrm{Co}$ & $7.31 \times 10^{-1}$ & \\
\hline${ }^{* 58} \mathrm{Co}$ & $1.97 \times 10^{-1}$ & \\
\hline
\end{tabular}

* Indicates elements that have two decay rates. 
irradiating lead. A few days after the cessation of irradiation, the activity in lead is dominated by ${ }^{200} \mathrm{Tl},{ }^{202} \mathrm{Tl}$, and ${ }^{203} \mathrm{~Pb}$. Only the 12 -day ${ }^{202} \mathrm{Tl}$ activity survives after the first week or two. ${ }^{6}$

\subsection{Spallation}

The production of ${ }^{54} \mathrm{Mn}$ discussed in the introduction may be described as neutron capture followed by the boiloff of several nucleons. The incident neutron energy required for reactions of this type can be quite low. In contrast, the production of isotopes with atomic masses far removed from those of the target nuclei involves a much greater nuclear disruption. Such spallation reactions usually have a higher threshold energy. For example, the induced $\gamma$ activity in a variety of elements for several incident proton energies is shown in Figs. II.9-II.12 of Ref. 20. At energies below $100 \mathrm{MeV}$ no significant long-lived activity is induced in calcium, while the picture has changed dramatically when the incident energy is $600 \mathrm{MeV}$ or above because of the production of ${ }^{22} \mathrm{Na}$.

About 2 stars/ $\mathrm{GeV}$ are produced in high-energy hadronic cascades. This implies a mean energy per collision of several hundred $\mathrm{MeV}$, well above energies at which spallation thresholds have significant effect on production rates. The same conclusion follows from the Moyer picture, in which the low-energy parts of the cascade are mainly propagated by neutrons in excess of $150 \mathrm{MeV}{ }^{21}$ While the assumption of a one-to-one relationship between star density and activation of these isotopes is still valid, one might expect that relatively more spallation products will be found nearer to the shower cores.

\subsection{Approximate Methods}

As has been noted, in an equilibrium situation the specific activity of an isotope is equal to its production rate. In turn, the production rate is proportional to the (local) beam particle loss rate. The scale factor relating energy loss in the cascade to the total activity immediately after turnoff (actually after 
$0.1 \mathrm{~h})$ is variously given as $1 \mathrm{~Bq} /(\mathrm{GeV} \mathrm{s})^{5}$ or $0.5 \mathrm{~Bq} /(\mathrm{GeV} \mathrm{s})^{22}$. Incident energies are not specified, but seem to be in the $200 \mathrm{GeV}$ range. The conversion is consistent with the result that about 2 stars $/ \mathrm{GeV}$ are produced in a cascade and Barbier's observation ${ }^{20}$ that one-third to one-half of all inelastic collisions (stars) result in radioisotopes whose lifetimes are between tens of minutes and several years.

\subsection{Input for Example Calculations}

As was mentioned above, the best that can be hoped for in calculating activities and dose levels in the present context is consistency within a factor of two. On the other hand, input numbers must of necessity come from a variety of sources, and, if they are to be compared, some uniformity between calculations must be achieved.

The examples discussed below have to do with long-lived activities in the SSC ring magnets resulting from distributed particle loss. Several months after turn-off, the total activity will have decreased by a factor of four, and the dominant residual activity will be ${ }^{54} \mathrm{Mn},{ }^{6}$ with a half-life of nearly a year. Other assumptions are as follows:

1. The proton loss rate is $2.3 \times 10^{8} \mathrm{~s}^{-1}$ in each ring. This is $\left(1-\mathrm{e}^{-1}\right)$ times the loss rate with $4 \times 10^{14}$ protons in each ring and a $300 \mathrm{~h}$ lifetime from beam-gas scattering.

2. The magnet may be modeled as an iron cylinder with radius $13.5 \mathrm{~cm}$. While this assumption ignores the ${ }^{60} \mathrm{Co}$ produced in the coils and stainless collars, these activities do not contribute substantially to surface dose.

3. Following Thomas and Stevenson ${ }^{5}$ (their Table 6.4), 2.25 stars $/ \mathrm{GeV}$ are assumed for $200 \mathrm{GeV}$ striking iron, or 450 stars per collision. The energy scaling from the table is such that 1.5 stars $/ \mathrm{GeV}$, or a total of 30,000 stars, would be expected in a $20 \mathrm{TeV}$ cascade. Van Ginneken's results using CASIM are substantially lower, with 20,000 or fewer per cascade. ${ }^{23}$ 
In using star density profiles from Ref. 23 , we therefore scale to a total of 30,000 stars for comparison purposes.

4. Gabriel and Santoro predict the production of $72{ }^{54} \mathrm{Mn}$ atoms when a $200 \mathrm{GeV}$ proton strikes an iron beam stop. ${ }^{6}$ If there are 450 stars and the inelastic $p$-Fe cross section (averaged over energy) is $703 \mathrm{mb},{ }^{24}$ the effective ${ }^{54} \mathrm{Mn}$ production cross section is $112 \mathrm{mb}$. Honda and Lal quote about a third of this value in the $500 \mathrm{MeV}$ to $700 \mathrm{MeV}$ energy range, ${ }^{7}$ but it has already been pointed out that (a) low-energy neutron production contributes substantially, and (b) the cross section is expected to be about $70 \mathrm{mb}$ at $50 \mathrm{MeV}$. We therefore assume that effectively $10 \%$ of all stars in iron produce ${ }^{54} \mathrm{Mn}$.

\subsection{A Worked Example: Activity in the Ring Magnets}

To illustrate the application of the methodology discussed above, we calculate the induced activity in the SSC ring magnets as the result of beam loss. Many of the input numbers come from Ref. 6, in which Gabriel and Santoro considered the effects of $200 \mathrm{GeV}$ protons striking an iron beam stop. Four days or so after the irradiation has ceased, the surface dose has decreased by a factor of two and about half of the remaining dose is due to ${ }^{54} \mathrm{Mn}$, with most of the balance due to ${ }^{48} \mathrm{~V}$. Since most of the isotopes of interest are somewhat similar $\gamma$ emitters, it may be assumed that surface dose behavior adequately mirrors radioactive isotope abundance inside the stop.

As stated in the last section, we assume that $2.3 \times 10^{8} 20 \mathrm{TeV}$ protons are lost per second around the ring, or $28 \mathrm{~cm}^{-1} \mathrm{~s}^{-1}$.

With our assumptions that $10 \%$ of 30,000 stars per cascade produce ${ }^{54} \mathrm{Mn}$ atoms, the production rate $R$ is $6.9 \times 10^{11}$ per second. The half-life of ${ }^{54} \mathrm{Mn}$ is $0.797 \mathrm{yr}$ (from Table 2.1), so $\tau=0.797 /(\ln 2) \mathrm{yr}=1.15 \mathrm{yr}$. From Eq. 2.1, the number of ${ }^{54} \mathrm{Mn}$ atoms present at times short compared with a year is $\tau R$, or $2.5 \times 10^{19}$ atoms. The disintegration rate is $R$, or $6.9 \times 10^{11} \mathrm{~Bq}=$ 
$19 \mathrm{Ci}$ per ring. Given the total magnet mass calculated from the parameters listed in the Conceptual Design Report ${ }^{1}\left(2.7 \times 10^{10} \mathrm{~g}\right)$, the average activation is $700 \mathrm{pCi}^{-1}$.

Alternatively, the rule of thumb given in the last section says

$$
\begin{aligned}
R & =1 \mathrm{~Bq} \mathrm{~s} \mathrm{GeV}-1 \times 20,000 \mathrm{GeV} \times 2.3 \cdot 10^{8} \mathrm{~s}^{-1} \\
& =4.6 \times 10^{12} \mathrm{~Bq}
\end{aligned}
$$

for the induced activity at the moment the machine is shut off. After a few months ${ }^{54} \mathrm{Mn}$ dominates and the activity has decreased by a factor of four, to $12 \times 10^{11} \mathrm{~Bq}$.

The two methods yield results which agree to within a factor of two, well within the uncertainties of the energy scaling of the first method and the approximate nature of the second. However, in the more careful first case the number of stars was assumed to increase by a factor of 67 when the energy scaled by a factor of 100 . In the second case application of the rule-of-thumb implicitly assumes that the number of stars scales linearly with energy. Had the same scaling been used in the second case, the result would have agreed to within $20 \%$.

\subsection{Absorbed Dose}

By "absorbed dose," one means the amount of energy absorbed by a unit test mass exposed to radiation. The composition of the test mass depends on the problem at hand, but if the concern is biological safety it should be tissue, or water. The conventional units for absorbed dose are rads, where 1 $\mathrm{rad}=100 \mathrm{ergs}^{-1}$, and grays, where 1 gray $=1 \mathrm{~J} \mathrm{~kg}^{-1}$, or 100 rads. For most applications the concept of "dose equivalent" is also of interest, where the dose equivalent is the absorbed dose multiplied by some quality factor which appropriately weights the effectiveness of the radiation in producing biological (or other) damage. In this report we are interested mainly in $\gamma$ - and 
$\beta$-radiation produced in radioactive decay, and for these radiations the quality factor is close to unity. We therefore limit the discussion to absorbed dose, with the understanding that in this context absorbed dose and dose equivalent are very nearly identical.

For the moment, consider a mass element in a uniformly activated uniform medium. In equilibrium (times long compared with the flight time of decay particles, or fractions of a nanosecond) each such element produces as much energy by radioactive decay as it absorbs. If the energy is emitted in the form of $\gamma$-rays, then ${ }^{5}$

$$
\dot{D}=2.13 A E_{\gamma},
$$

where $\dot{D}$ is the absorbed dose rate in rads $\mathrm{h}^{-1}, A$ is the specific activity in $\mu \mathrm{Ci}^{-1}$, and $E_{\gamma}$ is the emitted $\gamma$-ray energy in $\mathrm{MeV}$. The coefficient follows in making the appropriate unit conversions.

This result remains true for a finite object if the specific activity and medium are uniform for a few $\gamma$-ray absorption lengths from the fiducial mass. For $1 \mathrm{MeV} \gamma$-rays in iron, the absorption length is $2.0 \mathrm{~cm},{ }^{25}$ so the scale is 5 or $10 \mathrm{~cm}$.

In many cases of interest, it is not appropriate for fiducial mass to consist of the same material, but it should instead consist of a material such as organic tissue. In this case the coefficient must be corrected by the ratio of mass-energy attenuation coefficients in the host and sample materials. For $E_{\gamma} \approx 1 \mathrm{Mev}$ and most material combinations, the ratio is close to unity, but at lower energies the differences between e.g. iron and tissue are extreme. Since Compton-scattered $\gamma$-rays are involved in the radiation transport, we can expect a coefficient somewhat less than 2.13 even if $\sim 1 \mathrm{MeV}$ photons are emitted in the nuclear decay.

Now consider the fiducial mass as being on the plane interface between two uniform media. Both are of the same material, but only one is uniformly 
activated. By the symmetry of the problem the fiducial mass now absorbs exactly half the absorbed dose as before, and so experiences a dose rate

$$
\dot{D}=1.07 A E_{\gamma} .
$$

What if the unactivated part is now removed, so that we are considering the absorbed dose at the surface of a uniformly activated object? If the unactivated half is present, some small fraction of the dose absorbed in the fiducial mass arrives in the form of photons produced in the activated half but Comptonscattered in the unactivated half. Its removal therefore reduces the dose rate in the sample mass. Since only part of the Compton component is removed, the reduction is small, and the coefficient remains close to one. As before, the appropriate scale factor is the $\gamma$-ray attenuation length in the material: To evaluate the absorbed dose rate at the surface of an extended object whose dimensions are large compared with the attenuation length, we need only know the activity level in the vicinity of the measurement point.

It also follows that the absorbed dose at any distance from the surface of a uniformly activated thick slab of infinite extent is the same as that at the surface.

A final step permits extension of absorbed dose calculations to a wider class of geometries. We consider a small mass element (the "detector") some distance from the surface of the thick slab, and consider the flux of $\gamma$-rays due to the activity in a volume element $d V=r^{2} d r d \Omega$, at a slant distance $r$ and subtending a solid angle $d \Omega$ from the fiducial mass. Those $\gamma$-rays which originate in the volume and which are not absorbed or scattered in the intervening material contribute to the flux at the mass element. In addition, $\gamma$-rays produced elsewhere can Compton scatter in $d V$ to contribute to the flux. Finally, $\gamma$-rays emitted from $d V$ can Compton scatter elsewhere to reach the test mass from other directions. The flux from the direct process is proportional to $d V / 4 \pi r^{2}=d r d \Omega / 4 \pi$ times the appropriate attenuation factor. The same is 
true for Compton photons with a final scatter in $d V$, and very nearly true for photons produced in $d V$ which get to the test mass by scattering elsewhere, outside the cone defined by $d \Omega$. Integration over $r$ yields the flux from $d \Omega$; as before, most of it comes from the first interaction length near the surface. The picture which emerges from this approach is that the dose rate at a detector at any point can be calculated as the integral of surface dose rates over the solid angles subtended by the activated objects. The only additional approximation is that sizes of the objects and regions of activation are large compared with interaction lengths for the $\gamma$-rays in the material; near edges the equilibrium assumed between scatter-out and scatter-in Compton processes breaks down, just as it did when we removed the passive scatterer in the discussion of surface absorbed dose rate. As has been mentioned, "large" means large compared with the interaction length of the $\gamma$-rays, which is about $2 \mathrm{~cm}$ for ${ }^{54} \mathrm{Mn}$ and ${ }^{60} \mathrm{Co} \gamma$-rays in a ferrous material.

As an example, consider a uniformly activated slab of iron, $5 \mathrm{~cm} \times 2 \mathrm{~m}$ $\times 2 \mathrm{~m}, 20 \mathrm{~m}$ from the radiation detector. The surface dose at the center of slab's face is $\dot{D}_{s}$. When the detector is on the normal to the slab's face, $\Delta \Omega \approx(2 \times 2) / 20^{2}=0.01 \mathrm{sr}$, so $\dot{D}=(0.01 / 2 \pi) \dot{D}_{s}$. If the detector is $45^{\circ}$ from the normal, then $\dot{D}$ is reduced by $\sqrt{2}$, with some small increase because the edge subtends a finite solid angle.

Finally, consider an activated cylinder (e.g. an SSC dipole yoke) whose radius is large compared with a $\gamma$-ray interaction length. At its surface, the absorbed dose is the same as that near a flat slab with the same near-surface activation. As the detector is moved away from the cylinder, the flux drops as the solid angle subtended by the cylinder, or $\sin ^{-1} R / r$. For example, an SSC dipole yoke has a radius of about $13.5 \mathrm{~cm}$, while the radius of the cryostat is about $30 \mathrm{~cm}$. The absorbed dose at the cryostat is thus reduced from that at the surface of the yoke by a factor $\sin ^{-1} 13.5 / 30$, or 0.30 . 


\subsection{A Worked Example: Absorbed Dose at the Surface of an SSC Dipole}

We have already calculated the total ${ }^{54} \mathrm{Mn}$ activity in the $83 \mathrm{~km}$ ring to be $19 \mathrm{Ci}$, or $6.9 \times 10^{11} \mathrm{~Bq}$, or $8.3 \times 10^{4} \mathrm{~Bq} \mathrm{~cm}^{-1}$. To find the absorbed dose at the surface of the iron yoke $(r=13.5 \mathrm{~cm})$ or the (inside) surface of the cryostat, we need information about the distribution of the activity in radius. One source of such information is Fig. 23 from Ref. 23, reproduced as Fig. 2.3. The curves show the number of stars per radial increment (between $r$ and $r+d r$ ) in a solid iron cylinder, integrated over $z$. The dashed line is an estimated exponential fit to the small-radius part of the $20 \mathrm{TeV}$ curve; it is of the form $\mathrm{e}^{-r / \ell}$, with $\ell=10.5 \mathrm{~cm}$. With an intercept of $1700 \mathrm{stars} / \mathrm{cm}$ at $r=0$, the integral is 18,000 stars per $20 \mathrm{TeV}$ cascade, which, as we have mentioned, is a typical CASIM result.

However, only the slope is needed for the present purpose. For an activity profile of the form $b \mathrm{e}^{-r / \ell}$, the total activity is $b \ell$ and the activity density $A$ at radius $r$ is $b \mathrm{e}^{-r / \ell} / 2 \pi r$. With $b \ell=8.3 \times 10^{4} \mathrm{~Bq} \mathrm{~cm}^{-1}$,

$$
\begin{aligned}
A & =\frac{\left(8.3 \times 10^{4} \mathrm{~Bq} \mathrm{~cm}^{-1}\right) \times \mathrm{e}^{-13.5 / 10.5}}{(2 \pi \times 13.5 \mathrm{~cm}) \times 10.5 \mathrm{~cm}} \\
& =26 \mathrm{~Bq} \mathrm{~cm}^{-3} \\
& =8.9 \times 10^{-5} \mu \mathrm{Ci} \mathrm{g}^{-1}
\end{aligned}
$$

Since ${ }^{54} \mathrm{Mn}$ emits an $0.8 \mathrm{MeV} \gamma$-ray in the process of its $\mathrm{K}$-capture decay to ${ }^{54} \mathrm{Cr}$, Eq. 2.2 yields $0.076 \mathrm{mrad} \mathrm{h}^{-1}$. This result should be decreased by $10 \%$ or $20 \%$ to correct for the mass attenuation ratio between iron and tissue, and for the absence of backscattered Compton photons.

An alternative estimate can be made with the aid of the tables given in Ref. 6 , which give the calculated photon dose rate averaged over the surface of $2 \mathrm{~m}$ long iron cylinders of various radii when struck on-axis by $200 \mathrm{GeV}$ protons. When interpolated to $r=13.5 \mathrm{~cm}$, the average is $6.4 \times 10^{-10} \mathrm{rem} \mathrm{h}^{-1}$. The 


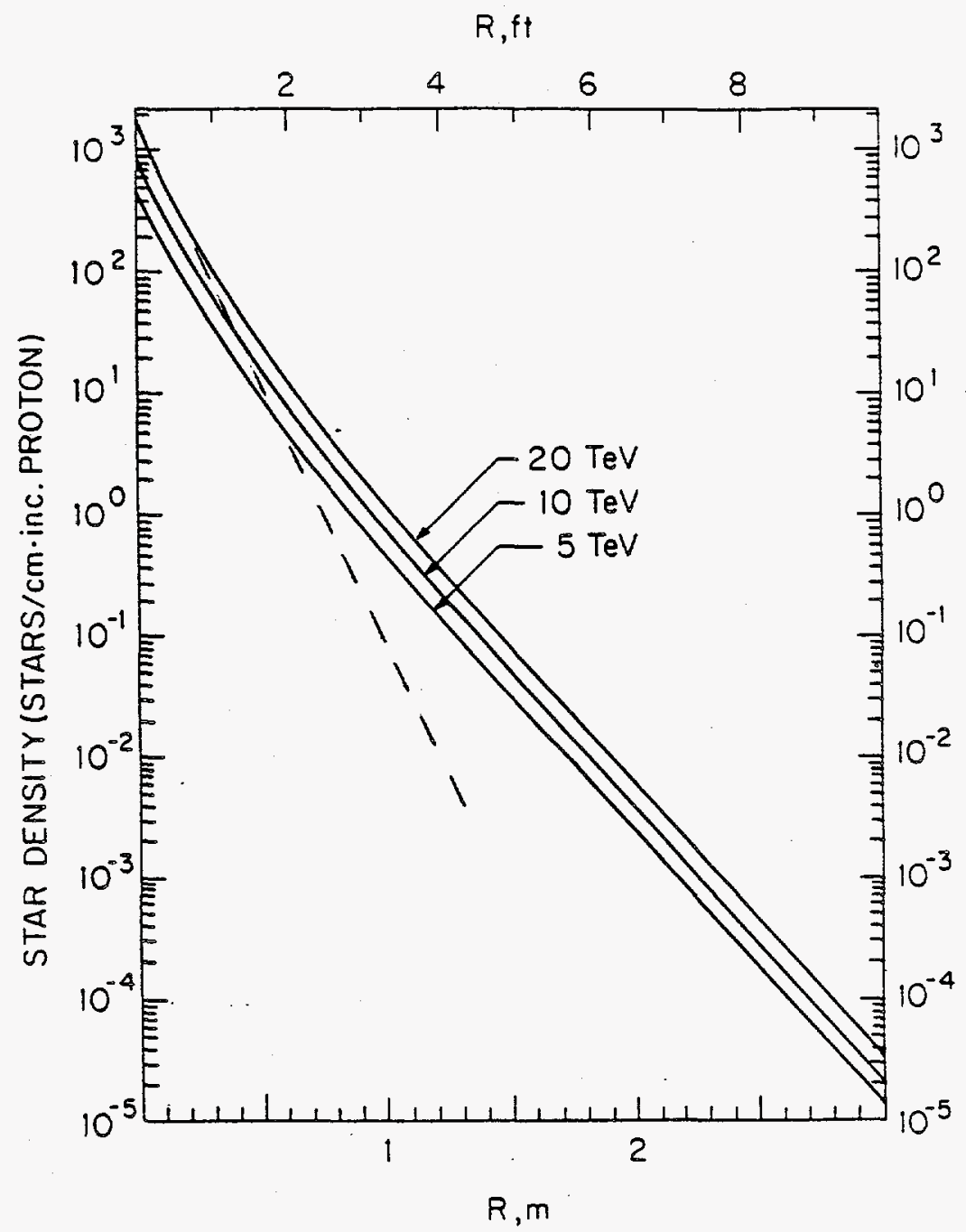

Figure 2.3. Longitudinally integrated star density (in stars/cm.incident proton) for 5,10 , and $20 \mathrm{TeV}$ protons incident on $5.0 \mathrm{~m}$ radius solid iron cylinder. The density is per unit width annulus at radius $r$. The calculation has a cut-off momentum of 0.3 $\mathrm{GeV} / \mathrm{c}$. Reproduced from Fig. 23 of Ref. 23. The dashed line $\exp (-r / 10.5 \mathrm{~cm})$ has been added.

average over the SSC's $83 \mathrm{~km}$ ring would be $2 / 83,000$ as large. Multiplying by the assumed loss rate of $2.3 \times 10^{8} \mathrm{~s}^{-1}$ and an energy scale factor of 67 , we obtain a surface dose rate of $0.24 \mathrm{mrad} \mathrm{h}^{-1}$ at machine turn-off, or 0.06 mrad $h^{-1}$ after four days. The agreement is fortuitous.

As has also been mentioned above, the absorbed dose at the cryostat wall $(r=30 \mathrm{~cm})$ will be lower by a factor of 0.30 , or $0.02 \mathrm{mrad} \mathrm{h}^{-1}$. 


\section{ABORT BEAM DUMPS}

In this section we focus on the activation and handling of the abort beam dumps for the SSC storage rings. ${ }^{26}$ Smaller beam dumps for the injector accelerators are also present in the SSC complex and are briefly described in Sec. 4.

There are two beam dumps for the storage rings, located as shown in Fig. 3.1. Beams in the SSC storage rings are stored for a time period of 12-24 hours. At the end of this time, the beams are kicked out of the storage rings into the abort dumps. The storage rings are then refilled and this cycle repeated. As stated in Sec. 1, an equivalent of 500 aborts of $4 \times 10^{14}$ protons at $20 \mathrm{TeV}$ may occur per year.

A schematic view of an abort beam dump for the SSC is shown in Fig. 3.2 and is conceptually similar, although larger in size, to beam dumps at many other accelerators. ${ }^{27}$ The beam incident on the dump is dispersed by magnets and/or other defocusing devices to decrease the energy density impinging on the dump. The beam energy $(1.2 \mathrm{GJ})$ is absorbed in graphite plates contained in a water-cooled aluminum structure. This structure is in turn surrounded by iron and by concrete. A waterproof layer surrounds the concrete. The dimensions of the iron and concrete are chosen so as to limit the activation of soil surrounding the dump-see Ref. 2 .

Graphite is chosen as the primary energy absorbing medium because it has a high melting point and because it has a low atomic number. The latter is important since the number of radioactive daughters with significant lifetimes produced in $p+C$ collisions is thereby constrained to a few species.

\subsection{Activation Levels in the Abort Dumps}

In order to estimate the activation of the dump, calculations with CASIM ${ }^{28}$ were used to estimate the number of stars produced per incident $20 \mathrm{TeV}$ proton. ${ }^{29}$ In the CASIM calculation the dump was assumed to be cylindrically symmetric. The incident beam strikes the dump in a uniform, circular spot $35 \mathrm{~cm}$ in radius. In our calculations of activation we assume that $2 \times 10^{17}$ protons/year at $20 \mathrm{TeV}$ are dumped for 25 years and take a cooldown period of six

months. Although substantial amounts of short-lived isotopes, such as ${ }^{11} \mathrm{C}$, are produced, 


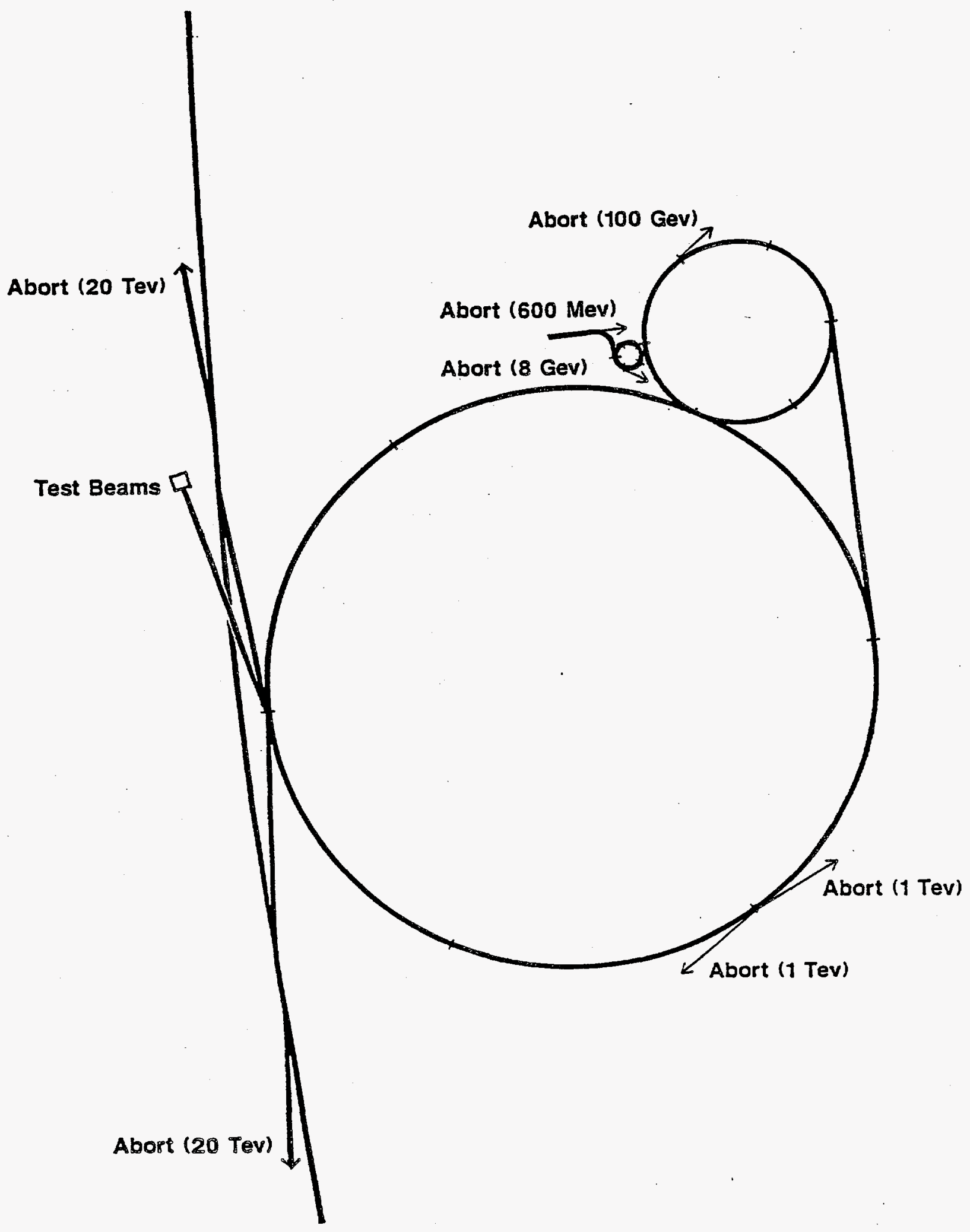

Fig. 3.1 An expanded view of the SSC injectors and abort region for the storage rings. 


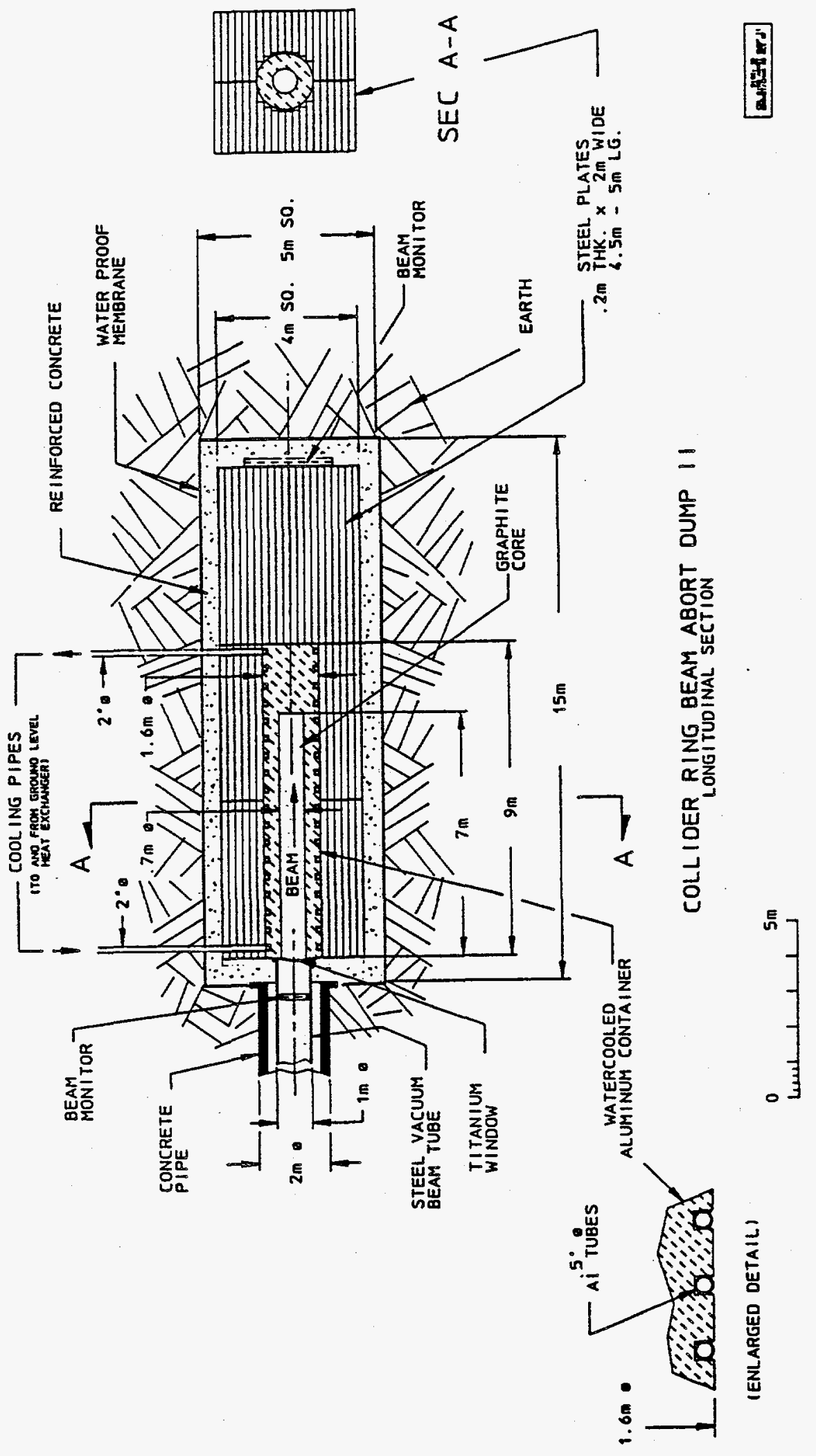

Fig. 3.2 A conceptual design of an abort dump for the SSC storage rings. 
within six months after shutdown only a few long-lived isotopes remain and are primarily responsible for the residual activation.

In the graphite there will be significant activation from the production of ${ }^{3} \mathrm{H}$ and $7 \mathrm{Be}$. In the aluminum ${ }^{22} \mathrm{Na},{ }^{3} \mathrm{H}$ and ${ }^{7} \mathrm{Be}$ will be present. In iron the situation is somewhat more complicated because of the large number of radioisotopes, which are produced in significant quantities. The most abundant, with half-lives greater than one day, are summarized in Table 3.1, along with those for other materials in the abort dump. However, some of the activity levels are misleading if taken at face value. For example, ${ }^{51} \mathrm{Cr}$ and the ground state of $52 \mathrm{Mn}$ are produced in a abundance ratio of about 3:1. The $\beta^{+}$decay of ${ }^{52} \mathrm{Mn}$ is followed by a cascade in which four $\gamma$-rays with a total energy of $3.96 \mathrm{MeV}$ are usually emitted, and in addition there are usually two $0.511 \mathrm{MeV} \gamma$-rays from the positron annihilation. ${ }^{51} \mathrm{Cr}$ decays by electron capture and produces a $0.310 \mathrm{MeV} \gamma$-ray 9 percent of the time. As a result, ${ }^{52} \mathrm{Mn}$ produces nearly 60 times the absorbed dose, as does the ${ }^{51} \mathrm{Cr}$ in spite of its lower production rate. It is thus so ineffective in producing detectable radiation that it could well be omitted from consideration.

In the concrete the total activation level will be very low, $\approx 30 \mathrm{pCi} / \mathrm{gm}$, comprised of a number of isotopes: ${ }^{3} \mathrm{H},{ }^{7} \mathrm{Be},{ }^{22} \mathrm{Na},{ }^{45} \mathrm{Ca},{ }^{54} \mathrm{Mn}, 55 \mathrm{Fe},{ }^{60} \mathrm{Co}$, and perhaps ${ }^{137} \mathrm{Cs}$ and ${ }^{152} \mathrm{Eu}$. The relative mix of these isotopes depends in detail on the composition of the concrete used. According to the 10 CFR 61 classifications of radioactive waste, ${ }^{30}$ all of the dump components would be low-level, Class A waste, taking the central core as a single component. 
TABLE 3.1. Activation in the storage ring abort dumps. (Only isotopes with half-lives longer than one day are included.)

\begin{tabular}{lllll}
\hline \hline & & & \multicolumn{3}{c}{ ACTIVITY (Ci) } \\
& NUCLIDE & HALF-LIFE & AT TURNOFF & AFTER 6 MONTHS \\
\hline \multirow{2}{*}{ Graphite } & ${ }^{3} \mathrm{H}$ & $12.3 \mathrm{y}$ & 111 & 108 \\
& ${ }^{7} \mathrm{Be}$ & $53.3 \mathrm{~d}$ & 83 & 8 \\
\hline \multirow{2}{*}{ Aluminum } & ${ }^{3} \mathrm{H}$ & $12.3 \mathrm{y}$ & 52 & 51 \\
& ${ }^{7} \mathrm{Be}$ & $53.3 \mathrm{~d}$ & 26 & 2.5 \\
& $22 \mathrm{Na}$ & $2.6 \mathrm{y}$ & 52 & 46 \\
\hline \multirow{2}{*}{ Iron } & $54 \mathrm{Mn}$ & $0.8 \mathrm{y}$ & 28 & 18 \\
& $51 \mathrm{Cr}$ & $27.7 \mathrm{~d}$ & 10 & 0.1 \\
& $52 \mathrm{Mn}$ & $5.6 \mathrm{~d}$ & 3.3 & - \\
& $48 \mathrm{~V}$ & $16.0 \mathrm{~d}$ & 3.0 & - \\
& $56 \mathrm{Co}$ & $0.22 \mathrm{y}$ & 0.6 & 0.12 \\
\hline
\end{tabular}

\subsection{Handling and Dismantling of the Abort Beam Dumps}

The core of the abort beam dump is envisioned to be one or more sealed cylinders (or boxes) of aluminum containing the graphite. The water-cooled aluminum cylinder is surrounded by a steel pile which is encased in a water-proof concrete skin. The weights of the various components are approximately:

\begin{tabular}{|c|c|}
\hline Material & Weight (metric tons) \\
\hline Graphite & 4.7 \\
\hline Aluminum & 34 \\
\hline Steel & 1650 \\
\hline Concrete & 350 \\
\hline & 2039 \\
\hline
\end{tabular}


The steel pile is envisaged to be made of 8 " cast plate. Using the formulae (in Ref. 27, adapted from Ref. 31; see also Appendix B) one may estimate the dose after a six month cooldown period at various locations within the dump. The results are summarized in Table 3.2. Doses are the maximum at the particular surface. These dose rates may be used to crudely estimate the man-rem burden upon disassembly of the dump.

TABLE 3.2. Surface doses in the storage ring abort dumps.

\begin{tabular}{ll}
\hline \hline \multicolumn{1}{c}{ Location } & Dose $(\mathrm{mrem} / \mathrm{hr})$ \\
\hline Outer surface of aluminum & 370 \\
Inner surface of iron & 1320 \\
Outer surface of iron & 0.2 \\
Iron - average & 50 \\
Concrete - average & 0.005 \\
Front face of aluminum & 25 \\
Back end of aluminum $(\mathrm{z}=970 \mathrm{~cm})$ & 620 \\
Front face, iron backstop $(\mathrm{z}=970 \mathrm{~cm})$ & 3305 \\
\hline
\end{tabular}

We envisage three possible modes of disposal of a dump:

1. Burial in situ.

2. Remove the graphite-aluminum core and leave the steel pile for future recycling.

3. Remove both the core and the steel pile.

In case 2 , the core would probably be removed from the upstream face of the steel pile. In case 3 , the core could also be removed from the upstream face or from above after unstacking part of the iron pile. If a use for the iron can be found - at another accelerator facility for example - then option 3 would be atractive.

In either case, we assume that the dump structure would be designed to allow minimum exposure and rapid dismantling of the most activated components, in particular the core. Only partial removal of the concrete and iron would be required to gain access to the core for remo- 
val from above or the core piece or pieces could be pulled out from the front of the dump. Much of this removal would be done by crane and hence the dose to workers would be small, resulting primarily from attachment of lifting bolts or fixtures. We estimate that removal of the core will require 2-5 days and that the total burden incurred would be less than $1 \mathrm{rem} .{ }^{32}$

Removal of the iron would likely require less than ten days, and the burden would be similar to removal of the core, less than $1 \mathrm{rem}$. Since the activation in the concrete is very small, no significant burden would be incurred in its removal. We estimate that the cost of removal of one dump would be approximately $\$ 60,000$.

Depending upon the characteristics of the SSC site, the dump may be left in place at the end of the useful life of the SSC. In this case the front face of the dump would be sealed with concrete. Since the dose at the front of the aluminum cylinder would be a maximum of $25 \mathrm{mrem} / \mathrm{hr}$, the burden incurred would be a few tens of mrem at most. Such a procedure would require 2-5 people working for less than one week.

\subsection{Disposal of Radioactive Water from the Abort Dump Cooling System}

The two abort dumps will be cooled with a closed loop water system consisting of coils wrapped around the aluminum cylinder (see Fig. 3.2). This water will become radioactive because of the interactions of the cascade with the water in the coils. The only long-lived isotope produced is tritium.

Using a specific model of the cooling system (see Appendix F), we find that there would be at most $0.14 \mathrm{Ci}$ of tritium in the water to dispose of at the time of SSC decommissioning. The concentration in the closed loop water volume $(1,600$ liters $)$ would be $84,000 \mathrm{pCi} / \mathrm{ml}$.

This is not a problem for disposal. It could be diluted into $6800 \mathrm{~m}^{3}$ of clean water and be below the EPA guideline of $20 \mathrm{pCi} / \mathrm{ml}$ for drinkable water. Alternatively, it could be used as a wetting agent for concrete, to solidify other low-level wastes. 


\section{ACTIVATION IN THE INJECTION SYSTEM}

The injection system for the SSC storage rings is shown in Fig. 3.1. The injection system is comprised of a $600 \mathrm{MeV}$ Linac, an $8 \mathrm{GeV} \mathrm{LEB}$, a $100 \mathrm{GeV} \mathrm{MEB}$, and a $1 \mathrm{TeV}$ HEB. The Linac and the LEB are very similar to the injection linac at Fermilab and to the Fermilab $8 \mathrm{GeV}$ booster, respectively. In its operational characteristics the MEB will be similar to the Fermilab main ring (conventional magnets) operating at $100 \mathrm{GeV}$. Finally, the magnets in the HEB are superconducting, and hence its operational characteristics will be very similar to the Fermilab Tevatron.

The areas within the injection complex which may become significantly activated are the injection/extraction points between the various accelerators and the dumps associated with each accelerator. Based upon Fermilab experience, ${ }^{33}$ we estimate that the exterior of most of the Linac will have a dose of $<1 \mathrm{mrem} / \mathrm{hr}$ on contact. Occasionally (every 2-3 years), access within the linac tanks will be required, in which case contact readings of $1 \mathrm{rem} / \mathrm{hr}$ may be encountered. The transfer line between the linac and the LEB will have "hot spots" with a dose of $20-40 \mathrm{mrem} / \mathrm{hr}$ at one foot. Since the Linac is a valuable and useful accelerator, experience would indicate that its components would be reused in other locations at the end of the useful life of the SSC, rather than being dismantled and stored.

The major points of activation in the LEB will again be in the extraction area from the LEB into the MEB and an internal beam dump. Based on the experience at Fermilab, ${ }^{34}$ the apparatus in these regions will become significantly activated, and maximum doses on contact will be $1-5 \mathrm{rem} / \mathrm{hr}$. The isotopes responsible for the long-lived activity will be predominantly ${ }^{54} \mathrm{Mn}$ and various cobalt isotopes. Previous experience with dismantling or repair activities at Fermilab indicate that a total burden of 100-500 mrem (shared among 5-10 people) would be incurred upon removal of these items (see Appendix C). Activation of the remainder of the magnets in the LEB or MEB will be small. 33 Doses on contact soon after shut-down would be 1-3 mrem/hr and this would decrease by a factor of about ten within a year. In the MEB, the 
areas of significant activation will be similar: the injection area from the LEB, the extraction area to the HEB, and the internal beam dump.

Since superconducting magnets will be used in the HEB except in the vicinity of the region for injection from the MEB, extraction into the storage rings or HEB abort dumps, and extraction for test beam targets, negligible activation of these magnets will occur. Activation and dose levels in the injection/extraction regions will likely be less than in the LEB or MEB$10-50 \mathrm{mrem} / \mathrm{hr}$ at one foot. 34

In the present design, two beam dumps would be necessary for the HEB. Two dumps would be needed since protons must be injected in opposite directions into the storage rings. The location of these dumps is shown in Fig. 3.1. Each dump will be very similar in scale to the existing beam dump at the Fermilab Tevatron. ${ }^{27}$ (See Figs. 4.1a and 4.1b) The Fermilab dump is designed for a maximum of $7 \times 10^{17}$ protons per year at $1 \mathrm{TeV}$. The HEB both injects protons into the storage rings and provides tests beams. We assume that a maximum of $10^{13}$ protons per spill are accelerated for both injection into the storage ring and for test beams. (In the present design a lower intensity, $10^{12}$, is envisaged during test beam use, but $10^{13}$ would be desirable). We furthermore assume 72,000 cycles per year for test beams and $28,000 \mathrm{cy}-$ cles per year for injection. Fermilab experience ${ }^{35}$ would indicate that approximately $10 \%$ of the cycles, or $10^{17}$ protons per year, might be aborted. The amount of beam aborted would be shared between the two dumps, with one dump receiving more beam during test beam operation. The activation in the dump may be crudely estimated by scaling from the $20 \mathrm{TeV}$ case described in Sec. 3.1. The scale factor is roughly $\left(\frac{1 \times 10^{17}}{2 \times 10^{17}}\right) \times\left(\frac{1}{20}\right)^{0.8}=0.05$. Hence, one would expect total activation levels in these dumps with values no more than $5 \%$ of the levels given in Table 3.1.

The HEB. will also be used to create low intensity test beams for studies of experimental apparatus. ${ }^{36}$ Beam extracted from the HEB will strike a target creating secondary beams. This procedure is similar to that now used to create secondary beams at Fermilab, which are used for testing apparatus and for physics experiments. The composition of a typical target 


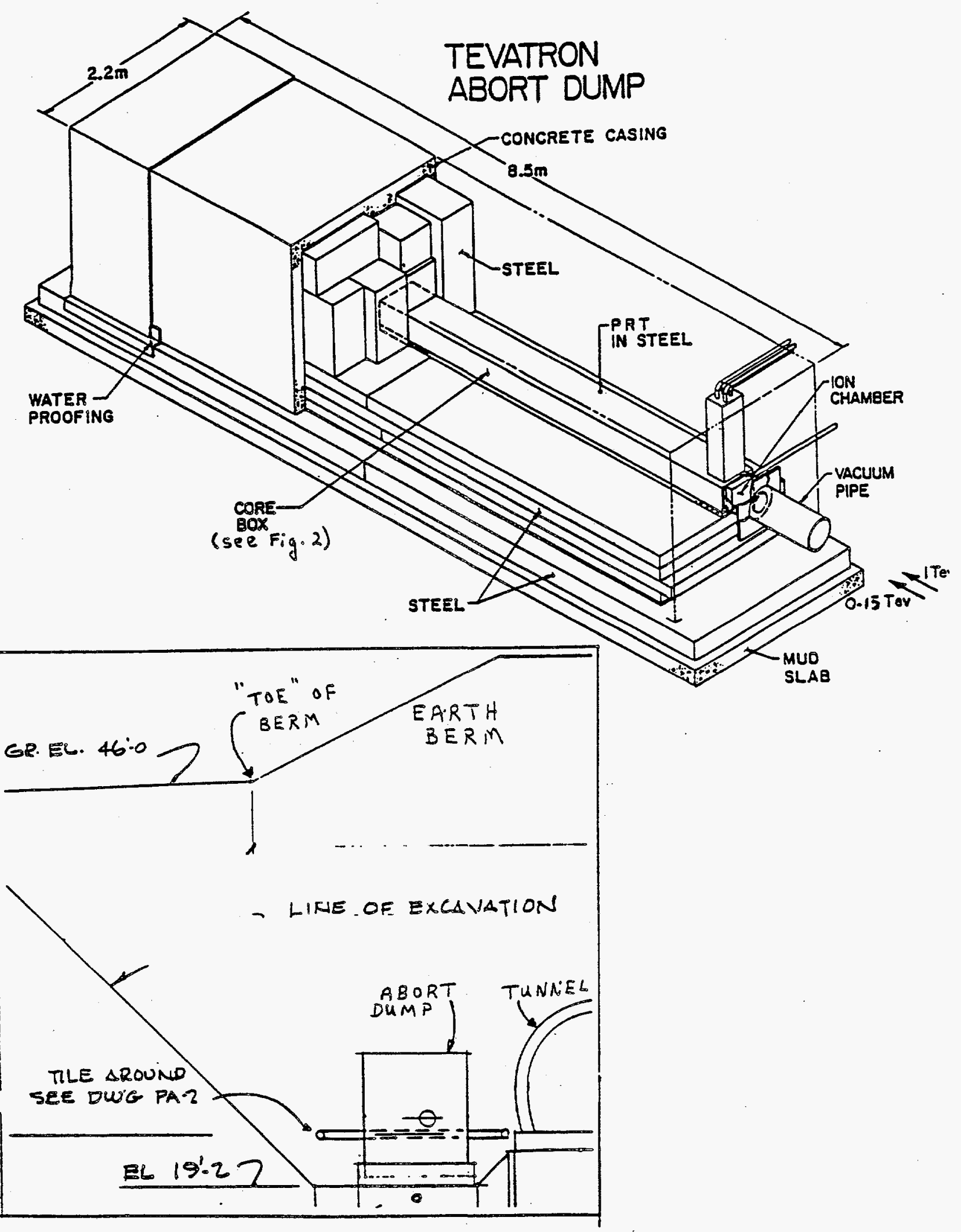

Fig. 4.1a Overall view of the Fermilab Tevatron abort dump. 


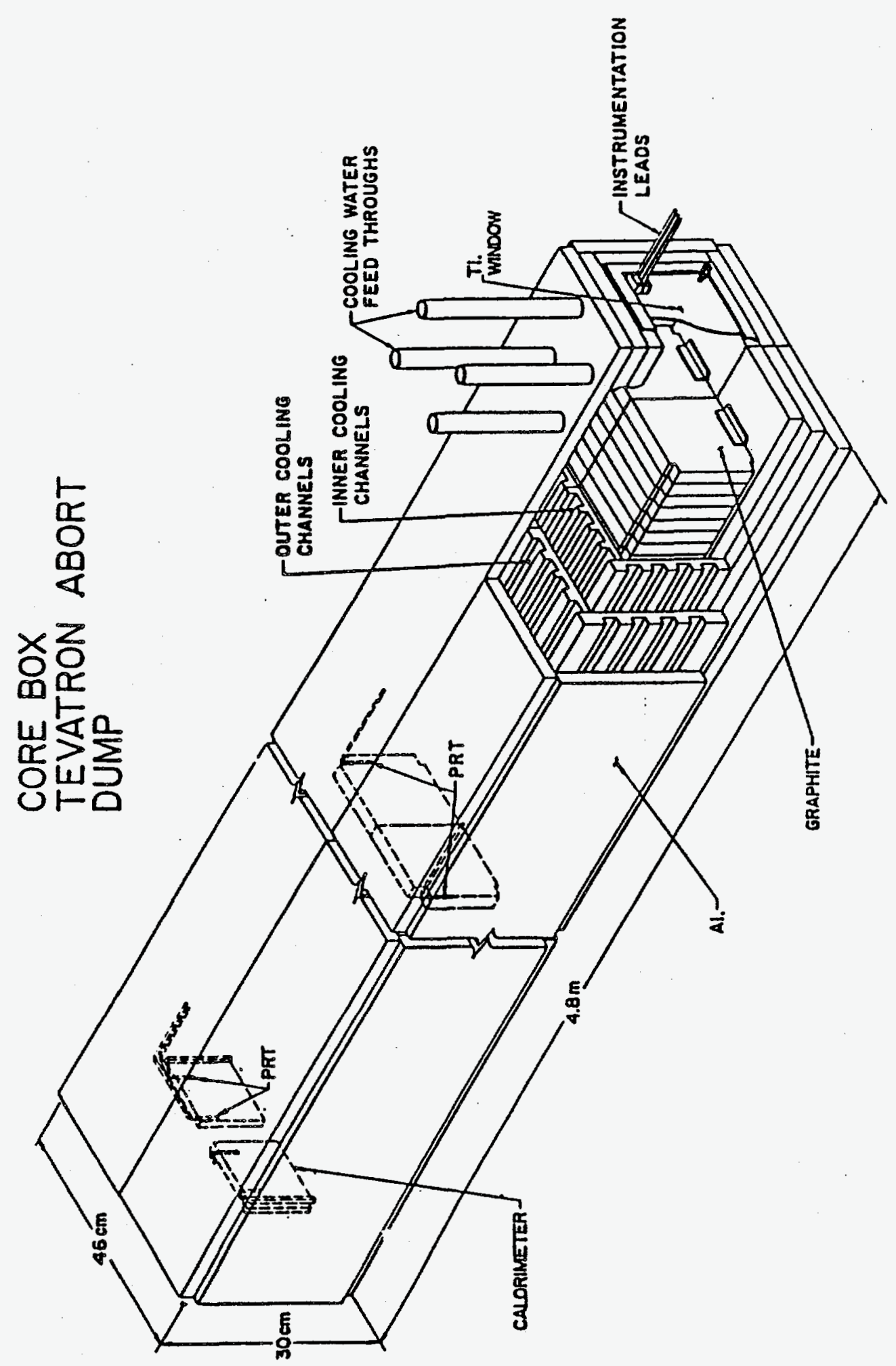

Fig. 4.1b The layout of the core of the dump. 
system is described in Appendix B. The target system contains a beryllium traget and magnets to select the appropriate secondary particles and to absorb the remnants of the primary beam. Four such systems may be used for this purpose at the SSC.

Activation levels and doses have been estimated for the Fermilab target systems. ${ }^{37}$ These levels would be similar at the SSC. Activation of the beryllium target just after turn-off will be approximately $0.15 \mathrm{Ci}$, primarily in ${ }^{7} \mathrm{Be}$. The total activation of the three magnets in the target system will be about $2 \mathrm{Ci}$, primarily ${ }^{54} \mathrm{Mn}$. Residual doses on contact in various regions of a target system are given below (refer to the drawing of the target system in Appendix B):

\section{DOSE ON CONTACT (MREM/HR)}

\section{LOCATION AT TURNOFF AFTER 6 MONTHS}

Front

Side/top near target

Side/top near dump

Back
1900

9.5

54

95
850

\section{0}

5

10

\section{BEAM LOSSES IN THE STORAGE RINGS AND AT THE INTERACTION REGIONS}

In this section we estimate the activation of the superconducting magnets in the storage rings, of the scrapers used to clean up the stored beam, of collimators near the interaction regions (IRs) and of possible detector components in or near the interaction regions.

Before beginning a discussion of the specific elements of the storage ring and interactions regions, it is worth noting that there are operational limits to beam losses in the storage rings at places other than the intersection points. With the assumptions given in Sec. 1, namely a maximum luminosity of $10^{34} \mathrm{~cm}^{-2} \mathrm{sec}^{-1}$ at an intersection region, approximately $3 \times 10^{9}$ protons per second would be lost through pp interactions at the collision points. Hence, during operation of the storage rings, losses elsewhere in the ring, other than from beam gas scattering, must be substantially less (at most $\approx 5 \times 10^{7}$ per second) than this rate in order to operate the machine for experiments. Thus, this provides an upper limit to loss of protons in the super- 
conducting magnets or in scrapers operating continuously during beam storage. Larger beam losses in scrapers may be tolerated intermittently (for a few seconds), for example, just after completion of injection into the storage ring. Prevention of quenches in the superconducting magnets also severely limit the number of protons that are allowed to be lost during beam injection and storage in the collider rings. This is described in the next paragraphs.

\subsection{Superconducting Magnets in the Storage Rings}

The superconducting magnets in the storage ring contain and focus the circulating proton beams. During normal operation in the storage ring mode, protons are lost from the circulating beam from interactions with residual gas around the ring and from pp interactions at the collision points. Most of the particles produced in the $\mathrm{pp}$ interactions at the collision points are absorbed by experimental apparatus, by collimators, or by scrapers at a few locations around the ring. Activation of these components is described in the sections below.

Most of the protons lost via beam-gas scattering are absorbed by the superconducting magnets, essentially in a uniform manner around the storage ring. The loss rate due to beam-gas scattering may be characterized by a lifetime, i.e., the time it takes to reduce the initial intensity by a factor of $\mathrm{e}^{-1}$. This lifetime due to beam-gas scattering is estimated to be 300 hours ${ }^{38}$ at an intensity of $4 \times 10^{14}$ protons. Given this lifetime, approximately $4.7 \times 10^{12}$ $\mathrm{GeV} / \mathrm{sec}$ is lost around the storage ring. Making the conservative assumption that this is deposited completely in the superconducting magnets (total mass $\approx 2.7 \times 10^{10}$ gms), the average total activity after a cooldown period of a few months would be approximately $700 \mathrm{pCi} / \mathrm{gm}$. The magnets are primarily composed of iron and copper. Therefore, the predominant long-lived isotopes responsible for the activity would be ${ }^{54} \mathrm{Mn}$ and ${ }^{60} \mathrm{Co}$, with small amounts of ${ }^{56} \mathrm{Co},{ }^{58} \mathrm{Co}$ and ${ }^{51} \mathrm{Cr}$. With the same assumptions, the average dose on contact with the outside surface of the magnet cryostat is estimated to be less than $0.02 \mathrm{mrem} / \mathrm{hr}$ a few months after shutdown.

A loss of approximately $1 \times 10^{6}$ protons $/ \mathrm{cm}-\mathrm{sec}$ or more into a superconducting magnet would be sufficient to cause a quench of the magnet, preventing operation of the storage ring 
for at least $2-3$ hours, the time required to refill the rings. Obviously such losses must be avoided and hence accidental beam losses will make a negligible contribution to the activation of the superconducting magnets.

Long-lived activation ( $>$ few days) of the concrete walls in the collider ring tunnels resulting from losses due to beam-gas scattering will be very small. The primary isotope of interest would be ${ }^{22} \mathrm{Na}\left(t_{1 / 2}=2.6\right.$ years). After a long irradiation time and a one day decay time, we estimate a surface dose on contact with the wall of $4 \times 10^{-4} \mathrm{mr} / \mathrm{hr}$.

\subsection{Beam Collimators and Scrapers}

There will be three types of collimators/scrapers in the SSC storage rings:

- protection collimators for the IR quadrupoles (see Sec. 5.3)

- abort channel collimators

- horizontal/vertical scrapers for clean-up of the tails of the beam and momentum scrapers to remove off-momentum particles

We describe the activation of the protection collimators for the IR quadrupoles in

Sec. 5.3, since these collimators may be strongly related to experimental apparatus in the interaction regions.

The location of the abort channel collimators is shown schematically in Fig. 5.1. These are fixed collimators. One of the collimators $(I)$ is located so as to prevent damage to magnets in the abort region in case of abort kicker misfire. The other collimator (II) prevents damage to downstream magnets during the normal abort sequence. The precise activation of these collimators is difficult to predict since it depends on the operational characteristics of the machine. However, we estimate that collimator I may absorb as much as $10^{15}$ protons per year at $20 \mathrm{TeV}$ and that collimator II might absorb $5 \times 10^{13}$ protons per year at $20 \mathrm{TeV}$ equivalent energy. Assuming that a collimator is an iron cylinder of radius $35 \mathrm{~cm}$ and length $200 \mathrm{~cm}$, it yields a total activation after a few month cooldown period of approximately $3 \mathrm{Ci}(500 \mathrm{nCi} / \mathrm{gm})$ for I and $0.15 \mathrm{Ci}(25 \mathrm{nCi} / \mathrm{gm})$ for II. The principal isotope responsible for the activity will be ${ }^{54} \mathrm{Mn}$. 
The probable location of the beam clean-up scrapers is shown in Fig. 5.2. In each ring there will be two pairs of scrapers to clean up in the horizontal and vertical planes and a pair to clean up off-energy particles. These scrapers will be adjustable. As noted in the introduction to the section, each scraper cannot remove more than $\approx 5 \times 10^{7}$ protons per second during continuous scraping or the luminosity lifetime will be seriously degraded. During the injection process, that is while the beam energy is $\approx 1 \mathrm{TeV}$, more beam may be scraped. For definiteness we assume that a scraper removes as many as $5 \times 10^{14}$ protons per year at $20 \mathrm{TeV}$ equivalent energy. The resulting total activation after a few month cooldown will therefore be approximately $1.5 \mathrm{Ci}$, again primarily from ${ }^{54} \mathrm{Mn}$ and possibly ${ }^{60} \mathrm{Co}$ if stainless steel is used for these scrapers.

In the vicinity of the beam clean-up scrapers and the abort channel collimators, the nearby concrete in the tunnel walls and nearby machine components (e.g., vacuum pipe, cables, etc.) may also become somewhat activated. An estimate of the activation in the concrete in the vicinity of a scraper may be obtained from the experience of decommissioning the $\mathrm{D} 0$ main ring abort at Fermilab. ${ }^{39}$ Their measurements of activation in the concrete tunnel walls near the D0 abort for the Fermilab main ring are shown in Table 5.1. Although the geometry of a scraper in the SSC will be somewhat different from the D0 abort, we may use these numbers to crudely estimate an approximate upper limit to the activity in the concrete walls near a scraper. In actuality the activation will very likely be less than our estimate since a beam that has been scraped will deposit energy over a larger region than a beam impinging on the D0 dump. Nevertheless, if we assume that $5 \times 10^{14}$ protons per year are scraped and that the geometry of the scrapers is similar to the $\mathrm{D} 0$ abort, then one would expect activation in the concrete at the level of approximately $75 \mathrm{pCi} / \mathrm{gm}$ of ${ }^{7} \mathrm{Be}, 300 \mathrm{pCi} / \mathrm{gm}$ of $22 \mathrm{Na}, 10 \mathrm{pCi} / \mathrm{gm}$ of ${ }^{54} \mathrm{Mn}, 10 \mathrm{pCi} / \mathrm{gm}$ of ${ }^{60} \mathrm{Co}$ and $15 \mathrm{pCi} / \mathrm{gm}$ of ${ }^{152} \mathrm{Eu}$. Activation of concrete in the vicinity of a thin internal target for a $500 \mathrm{GeV}$ beam has also been calculated by Gabriel, et al. 40 Extrapolating to the SSC would yield activation levels about twice those above, but these calculations neglect. 


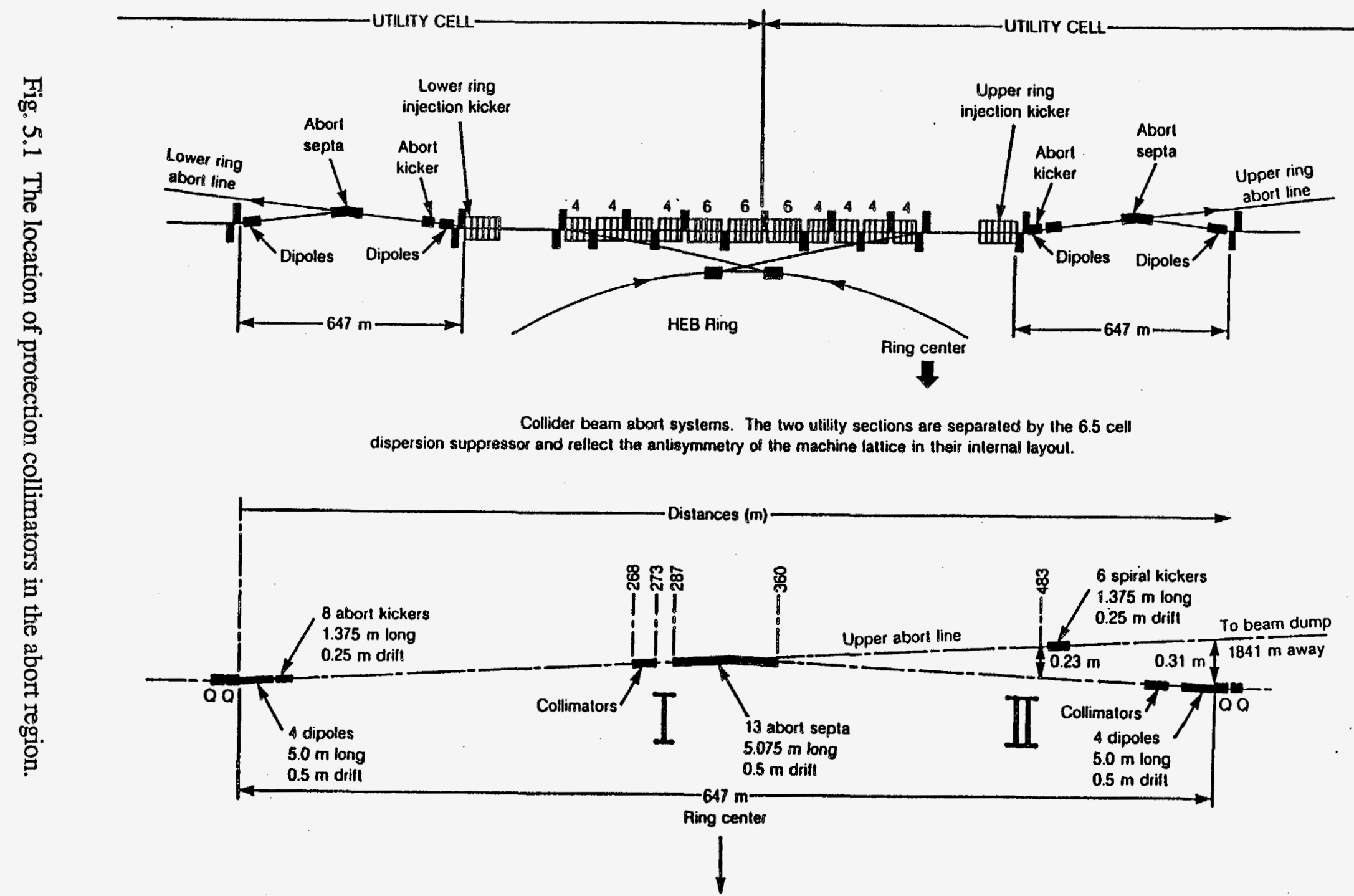




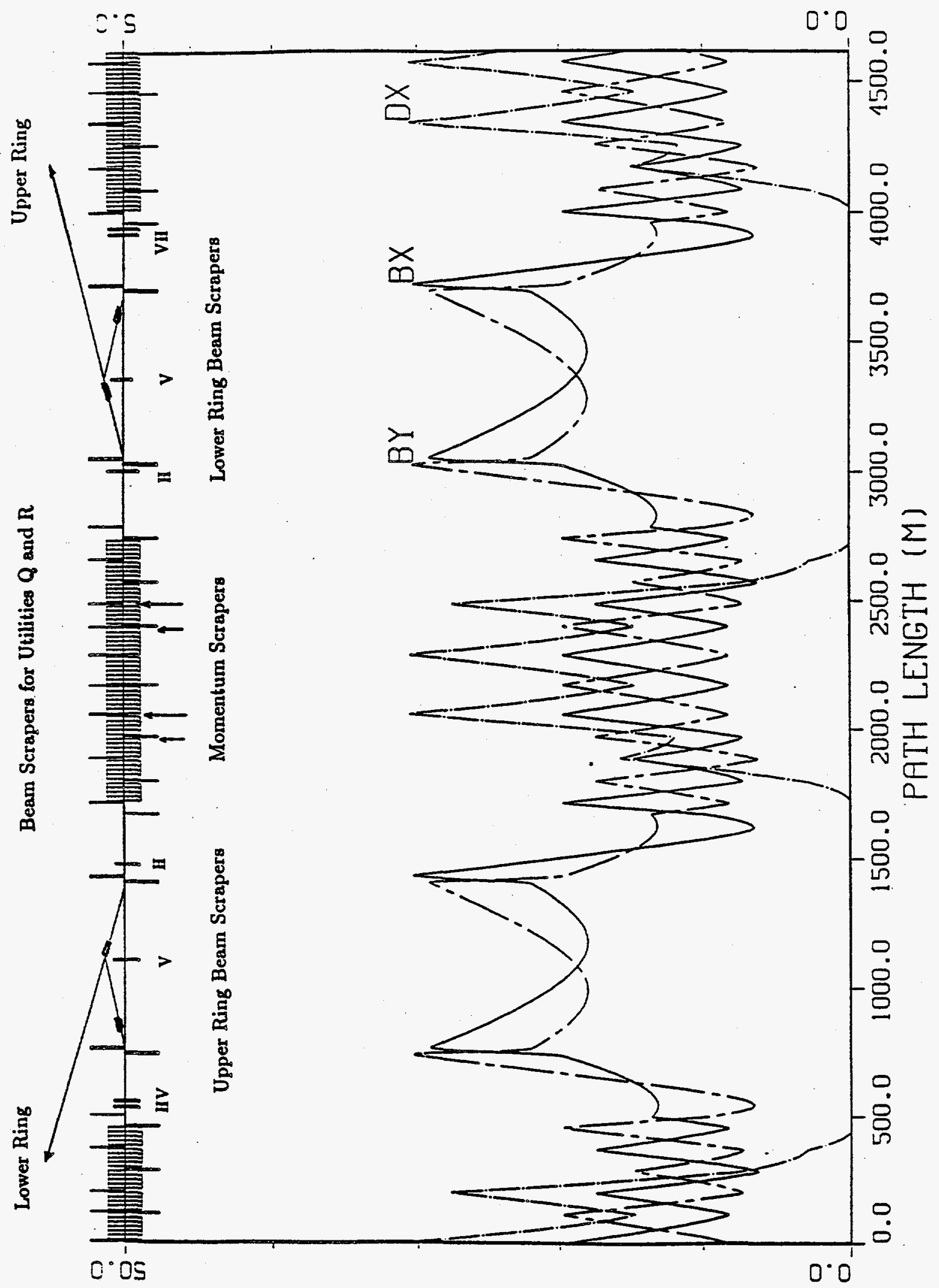

Fig. 5.2 The location of the scrapers. 
absorption in the scraper and nearby magnets. Gabriel, et al. also would indicate that other isotopes, ${ }^{3} \mathrm{H},{ }^{45} \mathrm{Ca}$ and ${ }^{55} \mathrm{Fe}$, would be present at levels comparable to ${ }^{22} \mathrm{Na}$.

TABLE 5.1 Measurements of activity in concrete near the Fermilab D0 internal abort dump.

\begin{tabular}{lll}
\hline \hline ISOTOPE & Av. CONCENTRATION & CURIES \\
\hline${ }^{7} \mathrm{Be}$ & $157.4 \mathrm{pCi} / \mathrm{gm}$ & $0.0600 \mathrm{Ci}$ \\
$22 \mathrm{Na}$ & 806.3 & 0.1920 \\
$54 \mathrm{Mn}$ & 25.1 & 0.0080 \\
$60 \mathrm{Co}$ & 21.4 & 0.0068 \\
$152 \mathrm{Eu}$ & 33.1 & 0.0105 \\
& & $0.2665 \mathrm{Ci}$ \\
\hline \hline
\end{tabular}

\subsection{Interaction Regions-mxperimental Apparatus}

The $\mathrm{pp}$ interactions at the intersection points produce a large number of secondary particles. For our purposes these interactions may be conveniently modeled by Monte Carlo programs such as ISAJET ${ }^{41}$ or PYTHIA ${ }^{42}$. We have used PYTHIA to estimate the average energy flow from charged particles per pp collision as a function of the polar angle with respect to the colliding beam axis (see Fig. 5.3). By far, most of the energy flow is near $0^{\circ}$, whereas very little energy, on average, flows into the larger angle regions. Hence residual activation of experimental apparatus will be confined to the region near $0^{\circ}$.

The layout of a high luminosity interaction region is shown in Fig. 5.4. A protection collimator is located in front of the first IR quadrupole to prevent the quadrupole from quenching. The other collimators are used to clean up the secondaries from the beam that pass through the first quadrupoles and from spray from the first collimator.

The protection collimator subtends an angular region from approximately 1 milliradians to 57 milliradians. An average of approximately $2500 \mathrm{GeV}$ per event is absorbed in this 
collimator. We assume an average of $10^{9}$ events $/ \mathrm{sec}$, ten times the present design value. Assuming this collimator is an iron cylinder $200 \mathrm{~cm}$ long and $35 \mathrm{~cm}$ in radius, it yields a total activation of approximately $2500 \mathrm{nCi} / \mathrm{gm}$ after a few month cooldown period. The surface dose would be approximately $750 \mathrm{mr} / \mathrm{hr}$. The other collimators in the IR region will be much less activated.

A side view of a typical large detector at the SSC is shown in Fig. 5.5. Such a detector is comprised of a large central component covering the angular region greater than about $5^{\circ}$, a forward piece covering $1^{\circ}$ to $5^{\circ}$, and the protection collimator. The protection collimator may in fact be an active device, a calorimeter, but the activation level will be similar to the iron cylinder case described above. Since such a detector is designed to absorb the energy emitted in the pp interactions over the angular region given, only an inner $\approx 1 \mathrm{~m}$ thick layer will undergo activation. No significant energy penetrates beyond this $\approx 1 \mathrm{~m}$ thick layer. Knowing the energy flow per event one may estimate the total activation after a few month cooldown period in the three angular regions as shown in Fig. 5.6. The central region of the detector will not be significantly activated. This is by far the most massive part of such a detector. The detector piece which subtends $\approx 1-5^{\circ}$, will be activated to approximately $6 \mathrm{nCi} / \mathrm{gm}$. These activation levels would be appropriate for iron, in which case the dominant isotopes will be ${ }^{54} \mathrm{Mn}$ and various cobalt isotopes. If the material is lead, which is more likely, the long-lived activity will be smaller, since the activation of lead results in isotopes such as $202 \mathrm{Tl}$ (12 day $1 / 2$-life). It is also possible that these detector components will be constructed primarily of depleted uranium. In this case the natural activity of the depleted uranium will be the dominating factor. 43

The experimental apparatus that will be present at the SSC will represent a very large investment in money (hundreds of millions of dollars) and in man-years. Previous experience, for example at the ISR (see Appendix D), would indicate that much of the apparatus would be salvaged for use in experiments at other accelerators. Hence after shutdown of the SSC, one may assume that most of the experimental apparatus would be removed for use at other accelerator or laboratory facilities. 


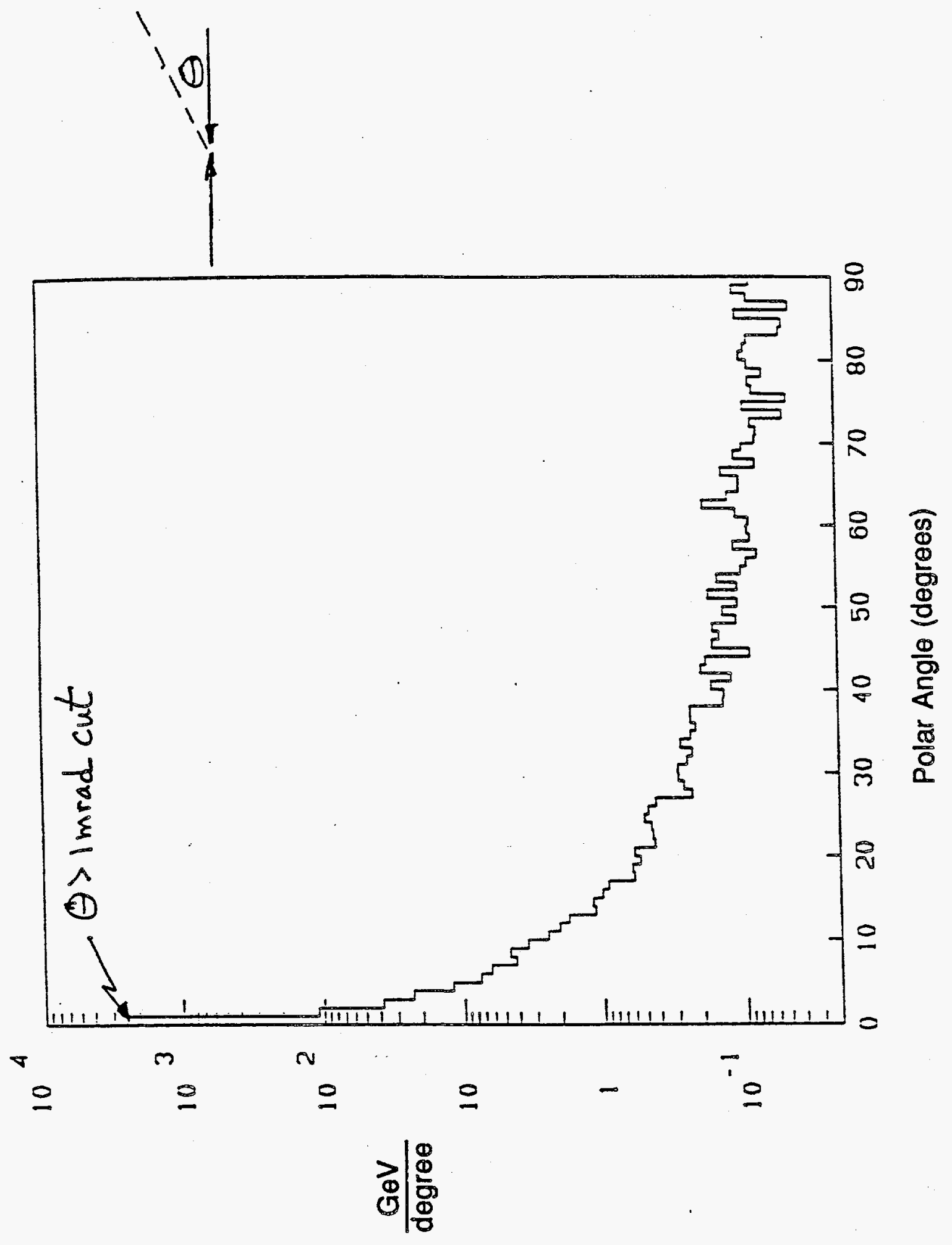

Fig. 5.3 The energy flow in GeV/degree vs. polar angle for the average pp collision at $40 \mathrm{TeV}$. 


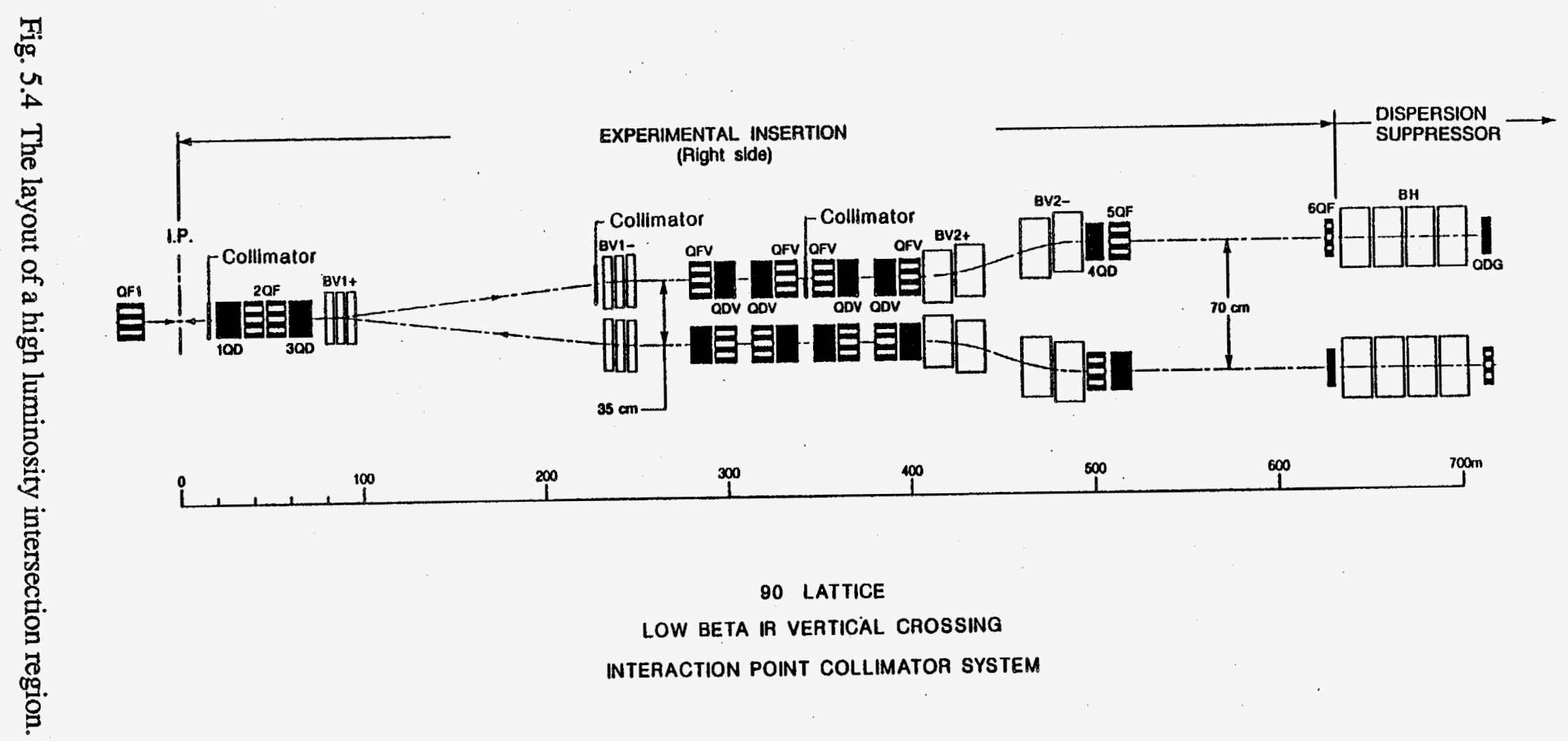




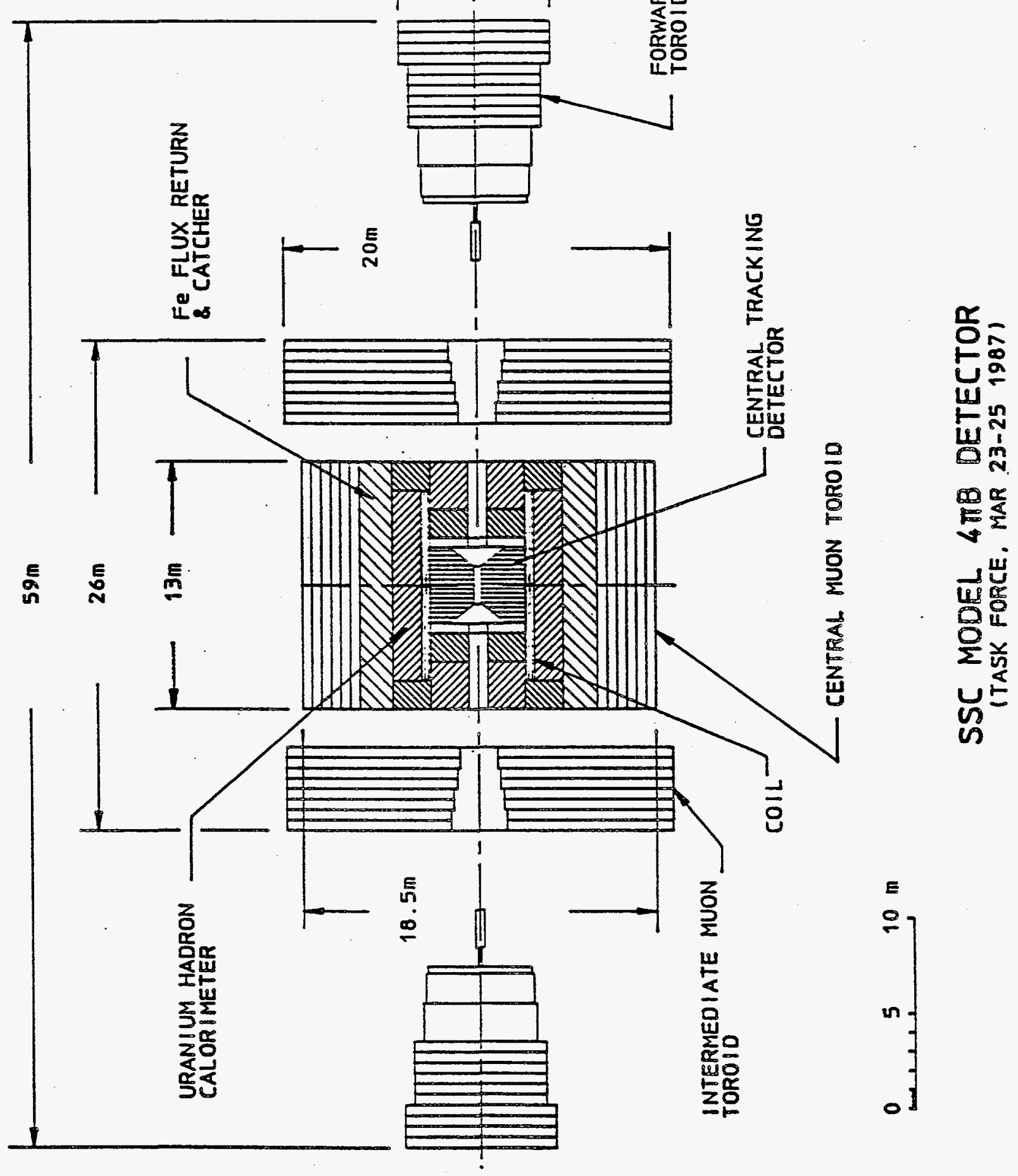

Fig. 5.5 A side view of a typical large detector for the SSC. 

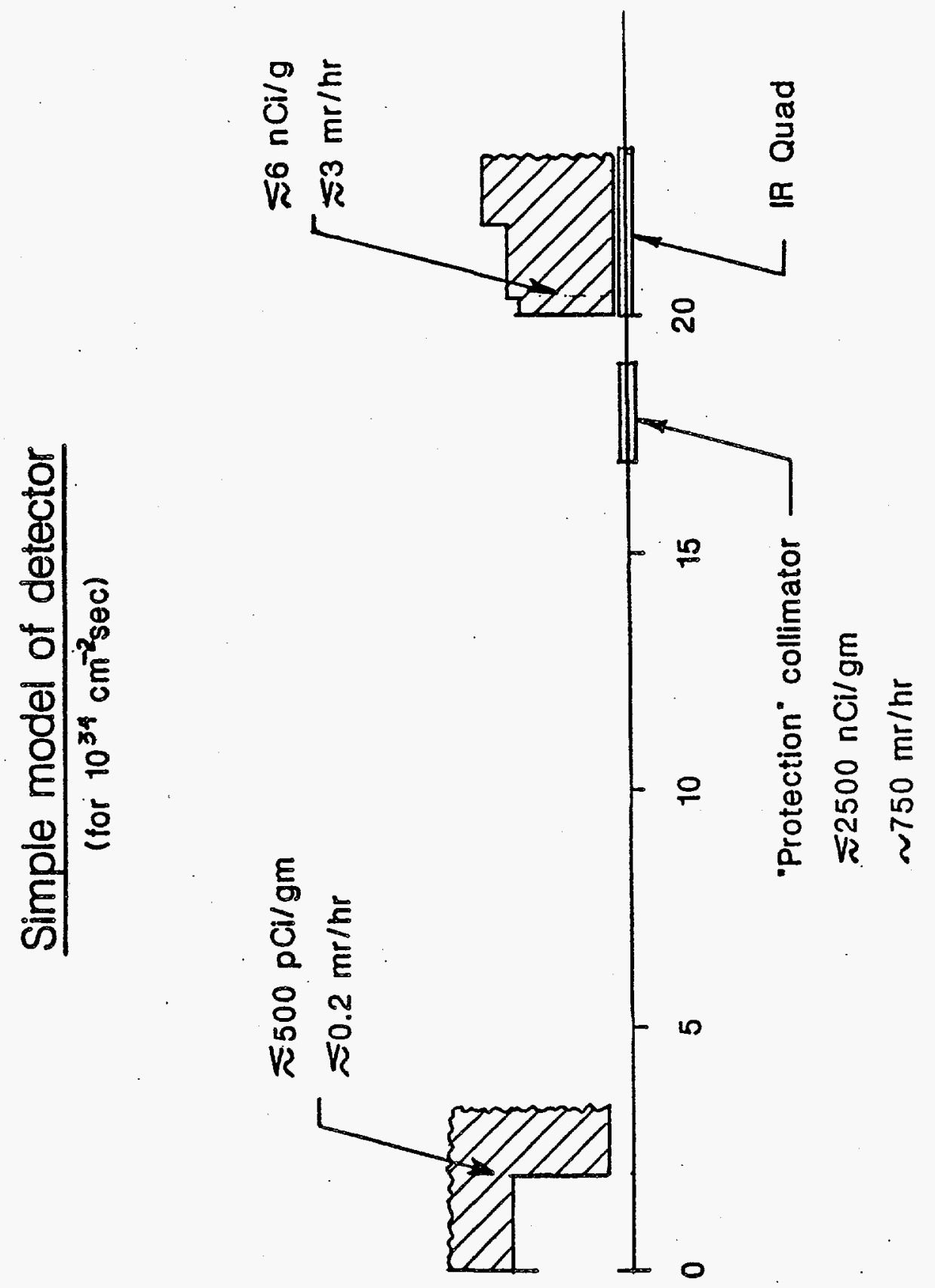

Fig. 5.6 The activation and dose in the relevant region of a large detector. 


\section{COMPARISON TO PREVIOUS DECOMMISSIONING EXPERIENCE}

A large number of accelerators have been decommissioned. This experience up to 1979 is described in Ref. 3. The decommissioning most relevant to the SSC is that of the Intersecting Storage Rings (ISR) at CERN which occurred in 1984 and 1985. The decommissioning process is described in more detail in Appendices D and E, and references therein. The ISR was also a pp collider, had a maximum center-of-mass energy of approximately $60 \mathrm{GeV}$, and was approximately $1 \mathrm{~km}$ in circumference. The magnets in the ISR rings were conventional, non-superconducting magnets. In each of the ISR storage rings, typical proton intensities of $5 \times 10^{14}$ protons were stored for a period of two to three days for physics experiments. The total number of protons per year injected into each of the ISR rings was as much as $2 \times 10^{17}$. These intensities are very similar to the maximum expected for the SSC, although of course the SSC is a larger and higher energy accelerator. Naively, if one imagined expanding the ISR to the size of the SSC, then the activity level would scale by the ratio of the energies to the power 0.8 and by their circumferences, i.e. by a factor of $\left(\frac{20000}{30}\right)^{0.8}\left(\frac{1}{85}\right)$, or about 2 . In actuality the proton loss rate in the SSC may be higher than in the ISR, because the ISR was built with a very large beam-tube aperture.

The ISR was decommissioned to provide storage and working space for the construction of the LEP $\mathrm{e}^{+} \mathrm{e}^{-}$storage rings at CERN. Many of the components of the ISR (magnets, etc.) were stored in one section of the large ISR "tunnel" (the ISR was housed in a very large tunnel, more like a circular hall ) and the remainder of the ISR "tunnel" was made available for working space with unrestricted access. The procedures to measure the activity levels during the decommissioning process and a summary of measurements are given in Appendix E. For the SSC, one would envision similar controls on the material removed from the SSC tunnels. Given the scale of the SSC, it seems unlikely that the all magnets would be moved as was the case in the ISR decommissioning. Much of the cost of decommissioning of the ISR was defrayed by the sale of salvageable components. A similar process is likely to occur for the 
SSC, although the quantity of salvagable items will obviously depend upon the nature of the decommissioning process for the SSC.

\section{SUMMARY}

In this report we have attempted to estimate the residual radioactivity levels that will be present after shutdown of the SSC accelerator and detector complex and have commented upon their disposition during the decommissioning phase of the SSC. We present a few general comments rather than review in detail the numerical estimates. Activation in the SSC complex will be similar to the levels present in existing large accelerator facilities at Fermilab and CERN. In terms of radioactivation, decommissioning of the SSC would not be qualitatively different than decommissioning of lower energy accelerators, in particular the CERN ISR.

\section{ACKNOWLEDGMENTS}

The members of Task Force would like to thank A. Van Ginneken, F. Turkot, R. Mau, P. Yurista, and S. Baker for providing much of the data used as input to the report. Discussions with $\mathrm{A}$. Gabriel were very useful in illuminating many aspects of the nature of radioactivation. Ed Wang provided the plot of energy flow in SSC events.

\section{REFERENCES}

1 The SSC is described in detail in Conceptual Design Report for the Superconducting Super Collider, edited by J. D. Jackson (March 1986).

2 Report of the Task Force on SSC Environmental Shielding, SSC-SR-1026 (July 1987).

3 J. H. Opelka, et al., Argonne National Laboratory Report, ANL/ES-82, 1979.

4 J. Bretka, P. J. Mathew, Health Physics, 48, 87 (1985); C. Papastefanou, et al., Health Physics, 47, 775 (1984).

5 R. H. Thomas and G. R. Stevenson, Radiological Safety Aspects of the Operation of Proton Accelerators, IAEA Technical Report Series (1987) (to be published). 
6 T. A. Gabriel and R. T. Santoro, Particle Accelerators 4, 169-186 (1973).

7 M. Honda and D. Lal, Phys. Rev. 118, 1618-25 (1960).

8 A. Van Ginneken, Fermi National Accelerator Laboratory Report FN-272 (Fermilab, Batavia, IL, 1975).

9 P. A. Aamio, J. Ranft, and G. R. Stevenson, "A Long Writeup of the FLUKA82 Program," CERN TIS Divisional Report TIS-RP/106-Rev (1984); P. A. Aarnio, A. Fassó, H-J. Moehring, J. Ranft, and G. R. Stevenson, CERN TIS-RP/168 (1986).

10 R. G. Alsmiller, Jr. et al., "Modifications of the High Energy Transport Code (HETC) and Comparisons with Experimental Results," presented at the ANS Topical Conference on Theory and Practices in Radiation Protection and Shielding (Knoxville, Tennessee, April 22-24, 1987); K. C. Chandler and T. W. Armstrong, "Operating Instructions for the High-Energy Nucleon-Meson Transport Code HETC," Oak Ridge National Laboratory Report ORNL-4744 (1972).

11 N. V. Mokhov, IHEP Preprint 82-168 (Serpukov, USSR, 1982); A. N. Kalinovsky, N. V. Mokhov, and Yu. P. Nikitin, "Penetration of High Energy Particles through Matter," (Energoatomizdat, Moscow, USSR, 1984).

12 M. B. Emmett, "The MORSE Monte Carlo Radiation Transport Code System," Oak Ridge National Laboratory Report ORNL-4972 (February 1975)。

13 R. W. Roussin, et al., "VITAMIN-E: A Coupled 174-Neutron, 38-Gamma-Ray Multigroup Cross-Section Library for Deriving Application-Dependent Working Libraries for Radiation Transport Calculations" (available from the Radiation Shielding Information Center, Engineering Physics and Mathematics Division, Oak Ridge National Laboratory, as DLC-113/VITAMIN-E).

14 T. A. Gabriel and R. T. Santoro, "Calculation of the Long-Lived Activity in Soil Produced by $500 \mathrm{GeV}$ Protons," Oak Ridge National Laboratory Report ORNL-TM-3262 (1970).

15 A. Van Ginneken and M. Awschalom, High Energy Particle Interactions in Large Targets, Vol. 1, Hadronic Cascades, Shielding, Energy Deposition (Fermilab, Batavia, IL, 1975).

16 T. A. Gabriel, R. G. Alsmiller, Jr., and J. Barish, Oak Ridge National Laboratory Report ORNL-4599 (1970). 
$17 \mathrm{~K}$. O'Brien, "Transverse Shielding Calculations for the Components of $\frac{1}{2} \mathrm{TeV}$ Proton Synchrotron," Health and Safety Laboratory, USAEC, Report HASL-199 (1968).

18 R. G. Alsmiller, Jr., and J. Barish, Nucl. Instrum. Methods 36, 309 (1965).

19 T. A. Gabriel, Nucl. Instrum. Methods 91, 67-72 (1971).

20 M. Barbier, Induced Radioactivity (North-Holland, Amsterdam, 1969).

21 B. J. Moyer, "Evaluation of Shielding Required for the Improved Bevatron," Lawrence Radiation Laboratory Report UCRL-9769 (June 1961); B. J. Moyer, in Premier Colloque International sur la Protection auprès des Grands Accélérateurs (Presses Universitaires de France, Paris, 1962) p. 61.

22 W. P. Swanson and R. H. Thomas in, Radiation Dosimetry, edited by K. R. Kase and B. Bjärngard,to be published by Academic Press (in preparation, 1987).

23 A. Van Ginneken, P. M. Yurista, and C. Yamaguchi, "Shielding Caluclations for Multi-TeV Hadron Colliders," Fermi National Accelerator Laboratory Report FN-447 (1987).

24 M. Aguilar-Benitez, et al., Particle Properties Data Book (North Holland, Amsterdam, April 1986).

$25 \mathrm{R}$. Thiesen and D. Vollath, Tables of X-ray Mass Attenuation Coefficients (Verlag Stahleisen, Düsseldorf, 1967); G. White Grodstein, "X-ray Attenuation Coefficients from $10 \mathrm{keV}$ to $100 \mathrm{MeV}$," Nat. Bur. Stand. (U.S.), Circ. 583 (1957); W. H. Tait, Radiation Detection (Butterworths, 1980).

26 See Ref. 1 for a more complete description of the beam abort systems including the dumps.

27 For example the abort dump at Fermilab, described in Fermilab TM-1196 by C. T. Murphy, F. Turkot and A. VanGinneken.

28 A. Van Ginneken, Fermilab Report FN-272 (1975).

29 The calculations for the SSC beam dump geometry were done by A. Van Ginneken.

30 Nuclear Regulatory Commission Report, 10 CFR 61, Ch. 1 (1-1-86 Edition).

31 M. Barbier, Induced Radioactivity (North-Holland, Amsterdam, 1969). (Also see ref. 27 for similar calculations for the Fermilab beam dump.)

32 See Appendix D for a compendium of so-called "hot jobs" at Fermilab.

33 P. Yurista, (Fermilab) (private communication). 
34 P. Yurista (private communication) (and presentations by J. D. Cossairt to the Task Force).

35 R. Mau (private communication).

36 For a description of a possible test beam layout see T. Kirk in Proceedings of the Summer Study of the Physics of the Superconducting Super Collider (Snowmass, Colorado, 1986). (Also see ref. 1.)

37 J. D. Cossairt, Fermilab TM 1168 (1983).

38 D. Bintinger (private communication).

39 P. Yurista (private communication).

40 T. A. Gabriel, R. G. Alsmiller, Jr., and J. Barish, ORNL-4599 (October 1970).

41 F. E. Paige and S. D. Protopopescu, in Proceedings of the UCLA Workshop on Observable Standard Model Physics at the SSC: "Monte Carlo Simulation and Detector Capabilities," edited by H-U. Bengtsson, C. Buchanan, T. Gottschalk and A. Soni (World Scientific Publishing, 1986).

42 H-U. Bengtsson, in Proceedings of the UCLA Workshop on Observable Standard Model Physics at the SSC: "Monte Carlo Simulation and Detector Capabilities," edited by H-U. Bengtsson, C. Buchanan, T. Gottschalk and A. Soni (World Scientific Publishing, 1986).

43 Depleted uranium is typically $99.8 \% 238 \mathrm{U}$. The remainder is $235 \mathrm{U}$ and trace amounts of other elements. ${ }^{238} \mathrm{U}$ decays via $\alpha$ emission with a half-life of about $5 \times 10^{9}$ years, yielding an activity of about $1.2 \times 10^{4} \mathrm{~Bq} / \mathrm{gm}$. The daughters, ${ }^{234} \mathrm{Th}$ and ${ }^{234} \mathrm{~Pa}$, decay via $\beta$ and $\gamma$ emission and are in radioactive equilibrium with ${ }^{238} \mathrm{U}$. Depleted uranium used in calorimeters is often clad, for example, in thin $(0.2 \mathrm{~mm})$ stainless steel which absorbs the $\alpha$ particles. Hence the dose from depleted uranium plates comes from $\beta$ and $\gamma$ emission. 


\section{APPENDIX A}

TASK FORCE ON RADIOACTIVATION

DR. J. DONALD COSSAIRT

FERMILAB, MS 219

P.O. BOX 500

BATAVIA, IL 60510

FNAL::93613

DR. GILBERT DROUET

SSC CENTRAL DESIGN GROUP

LAWRENCE BERKELEY LABORATORY

1 CYCLOTRON ROAD

BERKELEY, CA 94720

DR. KENNETH W. EDWARDS

SSC CENTRAL DESIGN GROUP

LAWRENCE BERKELEY LABORATORY

1 CYCLOTRON ROAD

BERKELEY, CA 94720

DR. MURDOCK G. D. GILCHRIESE

SSC CENTRAL DESIGN GROUP

LAWRENCE BERKELEY LABORATORY

$90-4040$

1 CYCLOTRON ROAD

BERKELEY, CA 94720

GILG@LBL

DR. DONALD E. GROOM

SSC CENTRAL DESIGN GROUP

LAWRENCE BERKELEY LABORATORY

$90-4040$

1 CYCLOTRON ROAD

BERKELEY, CA 94720

DEG@LBL
DR. C. THORNTON MURPHY

FERMILAB

MS 219

P.O. BOX 500

BATAVIA, IL 60510

THORNTON@FNAL

MR. KERAN O'BRIEN

P.O. BOX 967

SEDONA, AZ 86336

DR. S. PICHLER

CERN

1211 GENEVA 23

SWITZERLAND

DR. GRAHAM R. STEVENSON

CERN

1211 GENEVA 23

SWITZERLAND

GRS@CERNVM

DR. TIMOTHY E. TOOHIG

SSC CENTRAL DESIGN GROUP

LAWRENCE BERKELEY LABORATORY

$90-4040$

1 CYCLOTRON ROAD

BERKELEY, CA 94720 



\section{APPENDIX B \\ RESIDUAL RADIOACTIVITY MEASUREMENTS ALONG THE PB TARGET PILE}

J. D. Cossairt

September, 1987

In this brief note, measurements of residual radioactivity made along the PB target pile will be described. This was done to test the methods described by P. Gollon ${ }^{1}$ for predicting such radioactivity based upon surface star density calculations using CASIM ${ }^{2}$. These methods have been extensively used at Fermilab, especially in the design of the Tevatron II target piles. A design goal was to limit the maximum residual absorbed dose rates at "accessible" locations to $100 \mathrm{mrad} / \mathrm{hr}$ after long term irradiations and a one hour decay period. ${ }^{3}$

Estimating the residual absorbed dose rate for iron is especially troublesome using CASMM star density calculations because of the importance of the hadron (primarily neutron) flux produced below the calculational threshold of $300 \mathrm{MeV} / \mathrm{c}$ (47 MeV energy for nucleons). In the above reference, Gollon followed the general idea of the "danger parameter" devised originally by Barbier ${ }^{4}$ and wrote the following equation

$$
\mathrm{D}=\frac{\Omega}{4 \pi} \omega \mathrm{S}
$$

Here, $D$ is the residual absorbed dose rate due to a "semi-infinite" thick iron slab which has a surface star density rate $S$ ( the point of interest for determining the dose rate and hence, is $2 \pi$ for measurements "at contact." $\omega$ is the parameter determined by Gollon for two different irradiations

$$
\begin{gathered}
\omega=9 \times 10^{-3} \mathrm{mrad} / \mathrm{hr} \text { per star } \mathrm{cm}^{-3} \mathrm{~s}^{-1} \\
\text { for } \infty \text { irradiation time with } 0 \text { decay time } \\
\qquad \& \\
\omega=2.5 \times 10^{-3} \mathrm{mrad} / \mathrm{hr} \text { per star } \mathrm{cm}^{-3} \mathrm{~s}^{-1} \\
\text { for } 30 \text { days irradiation time with } 1 \text { day decay time }
\end{gathered}
$$

$\omega$ is thus proportional to Barbier's “danger parameter," $d$. Values for other irradiation and decay times not given by Gollon can thus be inferred by scaling. 
The PB target pile was chosen for study because it is a relatively "clean" geometry amenable to modeling with CASIM, as is seen in the vertical cross section shown here. This target pile was constructed of new iron shielding and was used for only some low intensity studies during the 1985 physics run. Thus, the present (1987) fixed target run is the "inaugural" run. In fact, over $1 / 2$ of the protons which have ever been delivered to this target were delivered during a 24 day period preceding these residual dose rate measurements. Thus, to fairly good approximation, after one day of decay (during a two day shutdown), it was possible to obtain results for a 30 day irradiation with one day cooldown and use one of the above $\omega$-values without adjustment. In fact, the week immediately preceding the residual absorbed dose rate measurements was exceptionally smooth with essentially no "downtime." During this 24 day period, the average rate of targeting was $1.35 \times 10^{10} \mathrm{~s}^{-1}$, if one only counts spills with beam in them. If one divides the total beam by the "real" time, a value of $7.21 \times 10^{9} \mathrm{~s}^{-1}$ is obtained. To make the comparison with measured residual absorbed dose rates, I chose the former value based upon the rather steady running during the final week.

CASIM calculations were made in the usual manner, taking care to model the magnetic fields in the target pile magnets. Yoke fields were modeled with analytical formulae which approximate the values of the components $\mathrm{B}_{\mathrm{x}}$ and $\mathrm{B}_{\mathrm{y}}$. The star density averaged over azimuth was then tabulated for the outer surface both longitudinally (as a function of $Z$ ) and on the front face of the pile (as a function of $\mathrm{R}$ ). The above formula was used to convert this to the residual absorbed dose rate using the stated proton intensity.

After a one day decay period, measurements of the absorbed dose rate were made using a standard Geiger-Müller survey instrument. It was found that for the longitudinal measurements, the dose rate at the walls was approximately equal, at contact with them, to that found at the target pile lateral surface. A distinct minimum absorbed dose rate, about 20 percent smaller than either contact reading, was found halfway between the pile and the more distant parallel wall. The activation of the wall is most likely due to the presence of ${ }^{24} \mathrm{Na}\left(\mathrm{t}_{1 / 2}=15 \mathrm{~h}\right)$. Therefore, the measured values on the longitudinal scan were divided by two for comparison 
with these calculations. No such adjustments were deemed necessary for the radial scan across the face of the target pile; no source of significant background upstream of the pile was found. No comparisons are possible downstream of the target pile because of the presence of activated components.

The comparison of the Monte-Carlo predictions with the measurements are shown in the figures. Across the face, the agreement is fairly good, especially given the sensitivity to upstream losses not considered in the calculations. The "humps" seen in the calculations are probably not real, but instead are an artifact of the statistical errors. In the longitudinal scan, the agreement is good in the middle of the pile but fails to "see" the sharp peak in the beam dump region within a factor of four or so. The last measurement point may, in fact be enhanced due the downstream activated components. Some of the "washout" of the predicted structure may, in fact, be due to the crude method of subtracting the wall background. Since the ${ }^{24} \mathrm{Na}$ is produced by thermal neutrons, some of the background peak due to the dump is likely to be spread over a larger region of the enclosure.

It is concluded that reasonable accuracy was achieved. It would be desirable to make future comparisons; in fact, a long term cooling curve at the end of the present fixed target run should be taken.

\section{REFERENCES}

1 P. J. Gollon, IEEE Trans. Nucl Sci. NS-23 \#4, 1395 (1976).

2 A. Van Ginneken and M. Awschalom, High Energy Particle Interactions in Large Targets, Volume 1 Hadronic Cascades, Shielding, Energy Deposition (Fermilab, 1975).

3 D. Cossairt (memo to K. Stanfield), "Report of the Target Pile Residual Dose Rate Committee" (members: S. Butala, D. Cossairt, R. Currier, D. Eartly, M. Gerardi, J. Lindberg, and R. Stefanski, May 31, 1983).

4 M. Barbier, Induced Radioactivity (North Holland, Amsterdam, 1979). 

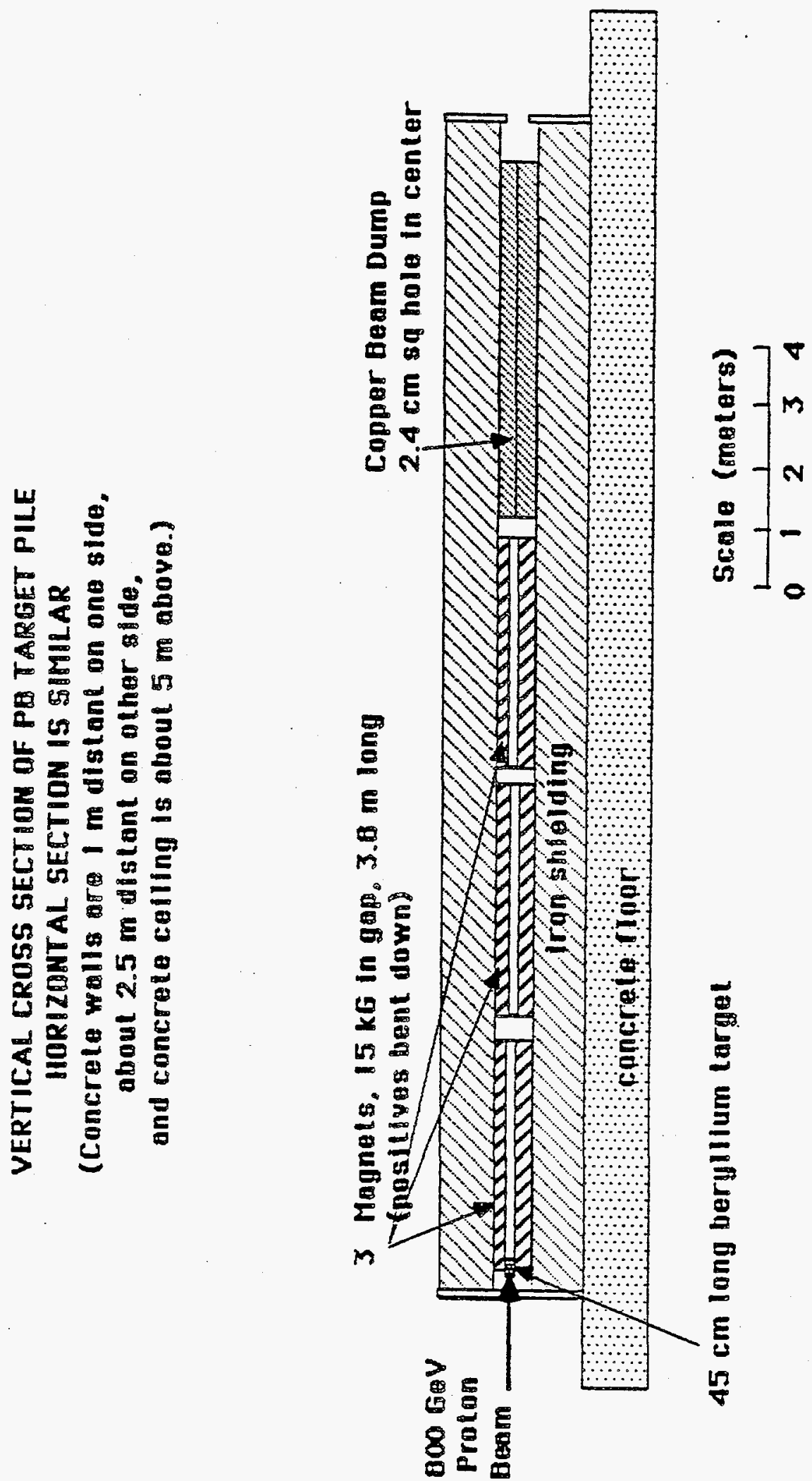

Fig. 1 The Fermilab target pile.. 


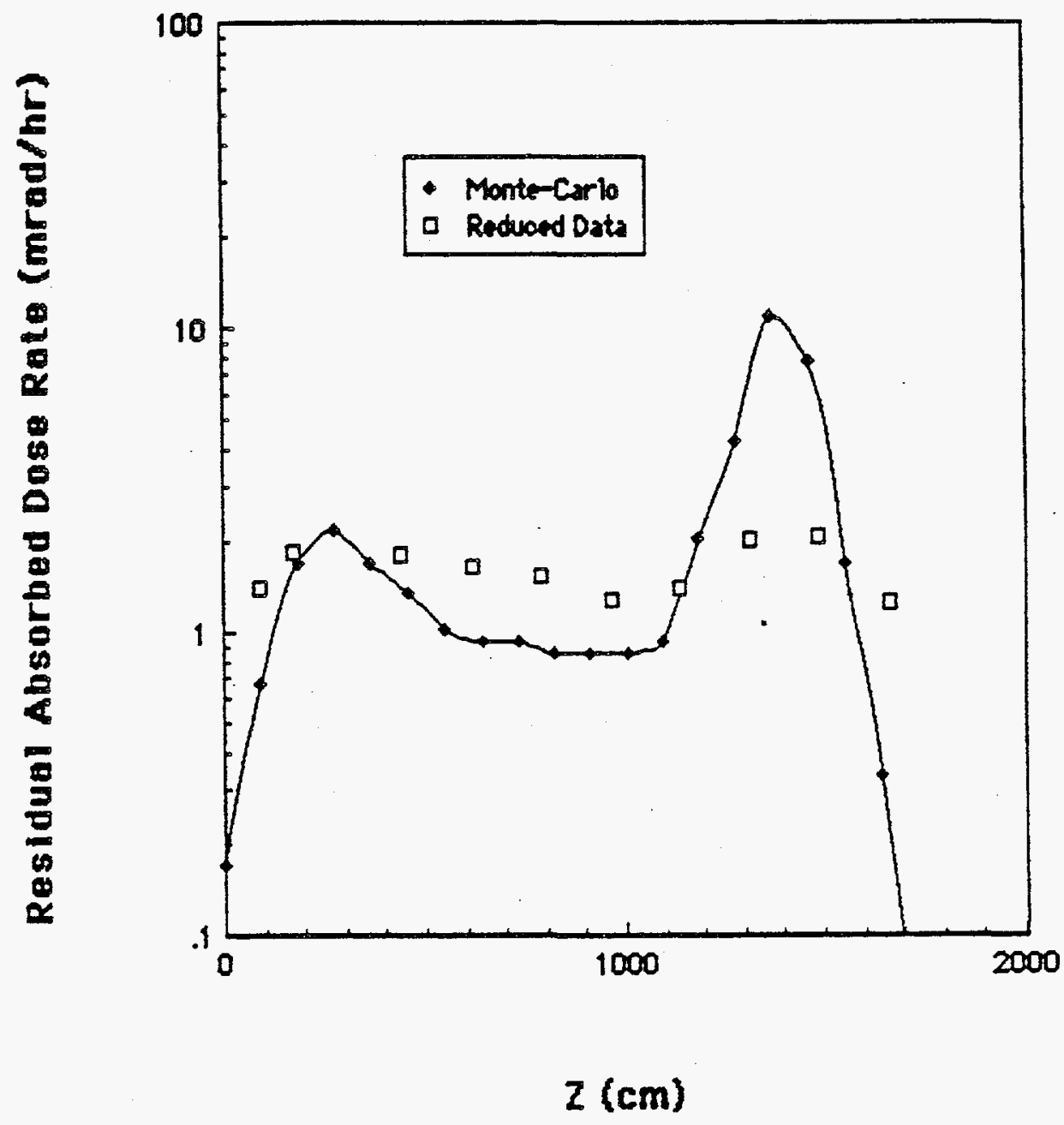

Fig. 2. Longitudinal survey of the target pile and comparison of measurements and predictions.. 


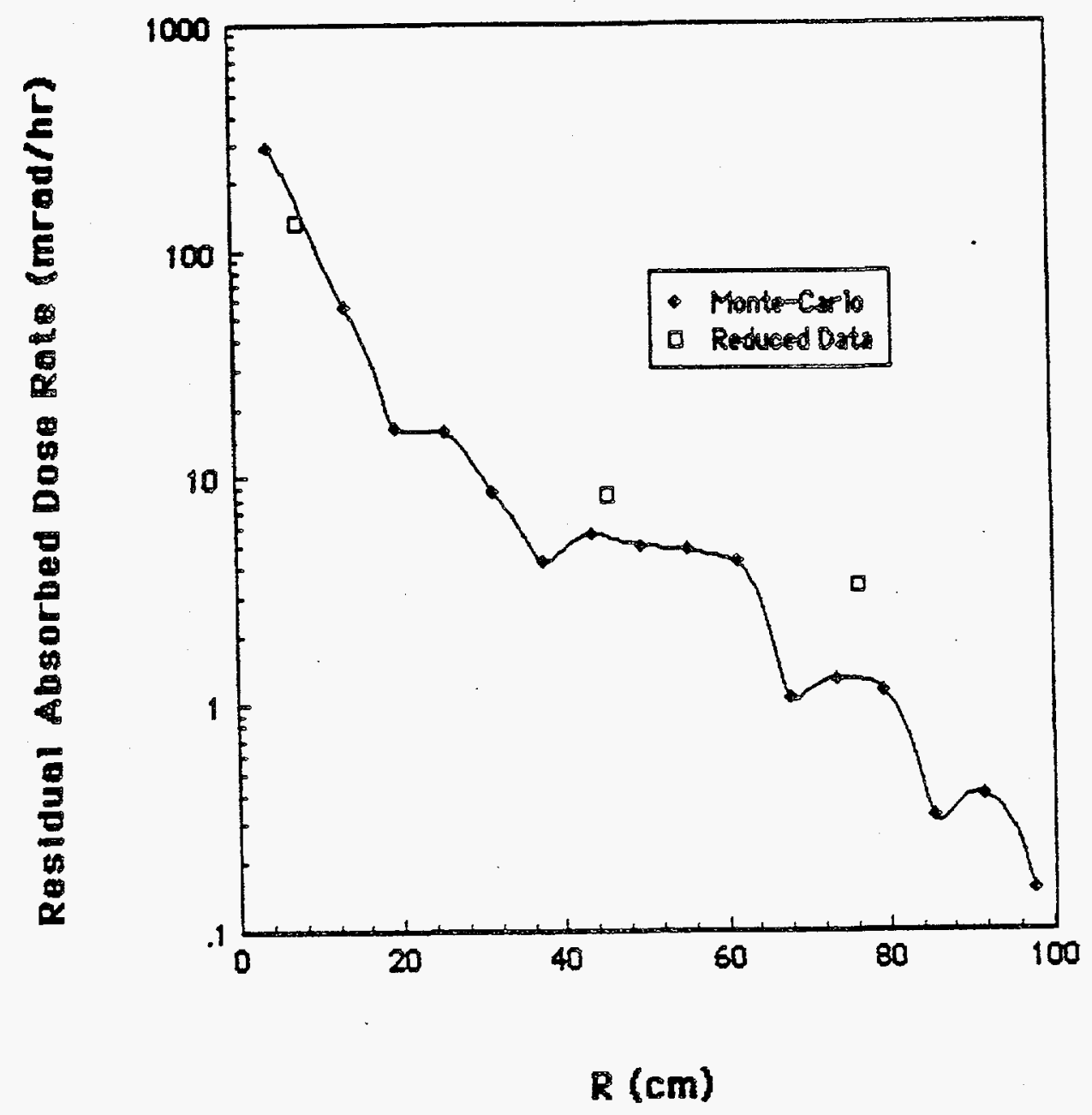

Fig. 3. Radial survey and comparison of measurements and prediction. 


\title{
APPENDIX C ANALYSIS OF EXPOSURE DUE TO WORK ON ACTIVATED COMPONENTS
}

\author{
J. D. Cossairt
}

September, 1987

In this brief note I summarize analysis of the exposure incurred in various maintenance jobs involving activated accelerator and beam line components. Because of their availability to me, most of these occurred in the fixed target areas of Fermilab. The following tabulation was made of the parameters associated with each job. After the table I list a brief description of the job. Thereafter, I present various plots of the various quantities in this table. All exposure rates are $\mathrm{mR} / \mathrm{hr}$ while all exposures accumulated are $\mathrm{mR}$. The exposure rates were generally measured at the Fermilab "standard" one foot distance from the activated component. Accumulated exposures are measured by self-reading pocket dosimeters.

\begin{tabular}{|c|c|c|c|c|c|}
\hline $\begin{array}{l}\text { JOB } \\
\text { NO }\end{array}$ & $\begin{array}{l}\text { MAXEXPOSURE } \\
\text { RATE }\end{array}$ & $\begin{array}{l}\text { NO. OF WORKERS } \\
\text { EXPOSED }\end{array}$ & $\begin{array}{l}\text { MAX INDIVIDUAL } \\
\text { EXPOSURE }\end{array}$ & $\begin{array}{l}\text { AV INDIVIDUAL } \\
\text { EXPOSURE }\end{array}$ & $\begin{array}{l}\text { TOTAL } \\
\text { EXPOSURE }\end{array}$ \\
\hline 1 & 1500 & 21 & 196 & 68 & 1441 \\
\hline 2 & 200 & 9 & 51 & 26 & 238 \\
\hline 3 & 600 & 12 & 110 & 35 & 420 \\
\hline 4 & 600 & 20 & 443 & 120 & 2385 \\
\hline 5 & 1000 & 20 & 307 & 103 & 2055 \\
\hline 6 & 1200 & 11 & 140 & 61 & 679 \\
\hline 7 & 1500 & 7 & 55 & 18 & 129 \\
\hline 8 & 30 & 6 & 34 & 16 & 96 \\
\hline 9 & 30 & 3 & 42 & 22 & 42 \\
\hline 10 & 250 & 7 & 50 & 20 & 50 \\
\hline 11 & 50 & 8 & 100 & 52 & 423 \\
\hline 12 & 50 & 10 & 50 & 13 & 131 \\
\hline 13 & 50 & 6 & 25 & 18 & 105 \\
\hline 14 & 30 & 6 & 40 & 28 & 169 \\
\hline
\end{tabular}




\section{Brief Descriptions of the Jobs Listed Above}

1. Repair of a water leak in a "target box" cooling loop assembly just downstream of heavily irradiated beam dump

2. Replacement of a failed magnet downstream of a heavily irradiated beam dump

3. Changeover in the sweeping magnets in a target box to allow a different running mode

4. Rework of the MPO1 location in the Booster (courtesy of P. Yurista)

5. Repair of the same water leak as No. 1 at a different time

6. Salvage and reclamation operations on a target train

7. Insertion of a pin in a moving target holder on a target train. Finger and hand doses are not included in these totals

8. Replacement of Kautzky valved downstream of a heavily irradiated beam dump

9. Torch-cutting of alignment tracks within a target pile

10. Repair of a target box magnet

11. Removal of target box "drawers" from a storage "cave"

12. Repair of magnet just upstream of a primary proton target

13. Repair of "closed loop" water system

14. Salvaging of bedplate from a target train 


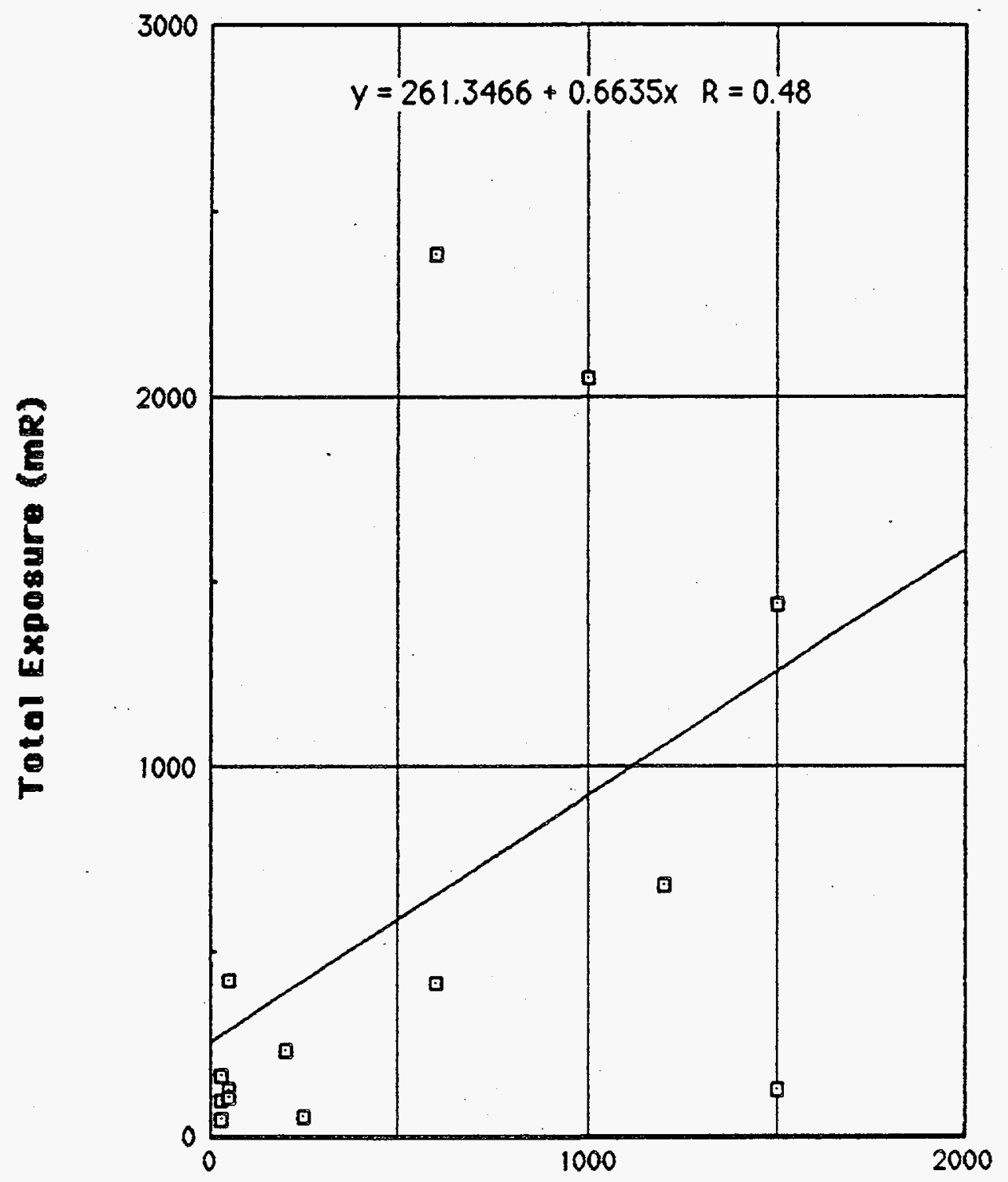

Peak Exposure Rate (mR/hr)

Fig. 1. Total exposure as a function of peak exposure rate. 


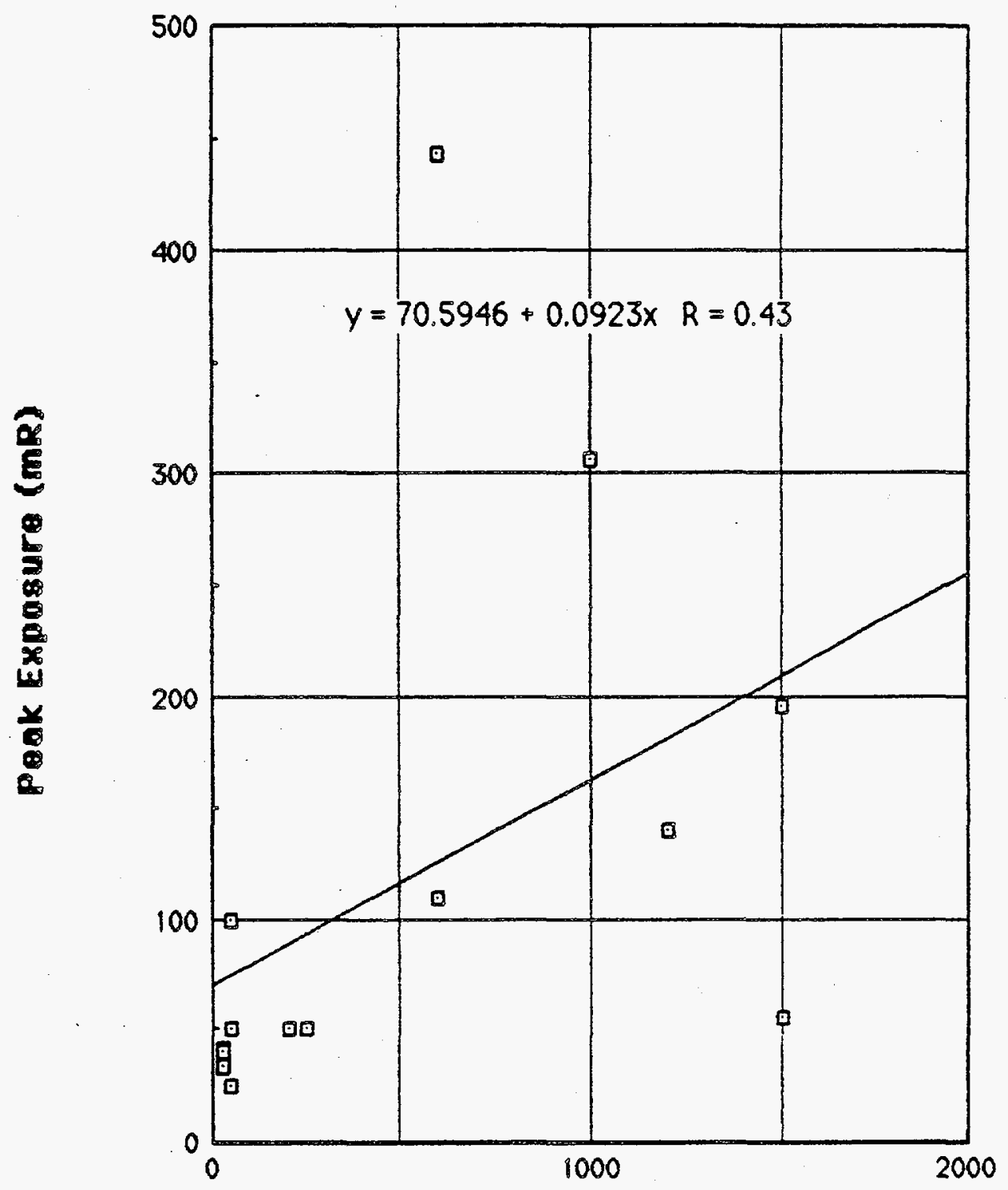

Peak Exposure Rate (mR/hr)

Fig. 2. Peak exposure as a function of peak exposure rate. 


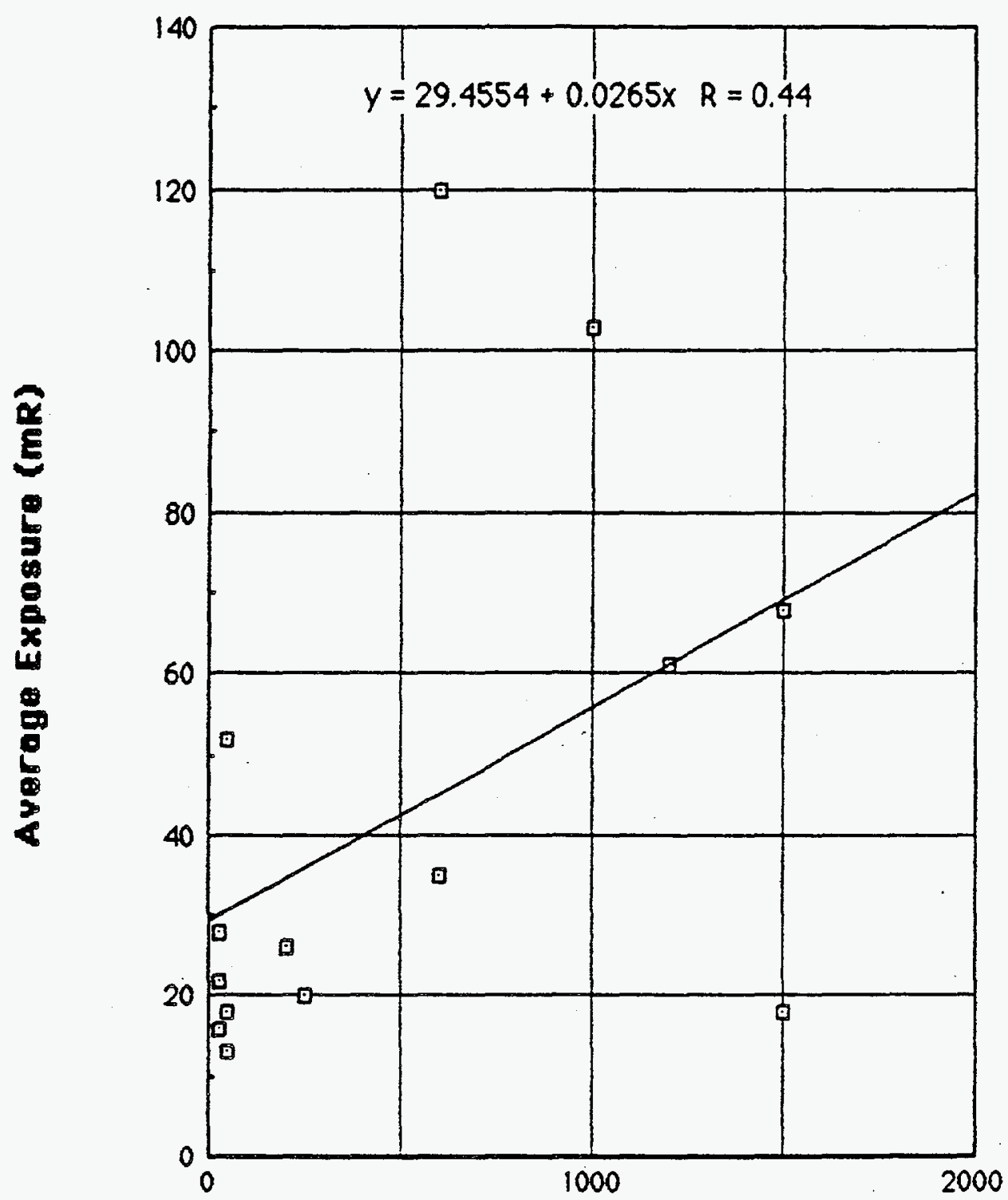

Peak Exposure Rate (mR/hr)

Fig. 3. Average exposure as a function of peak exposure rate. 


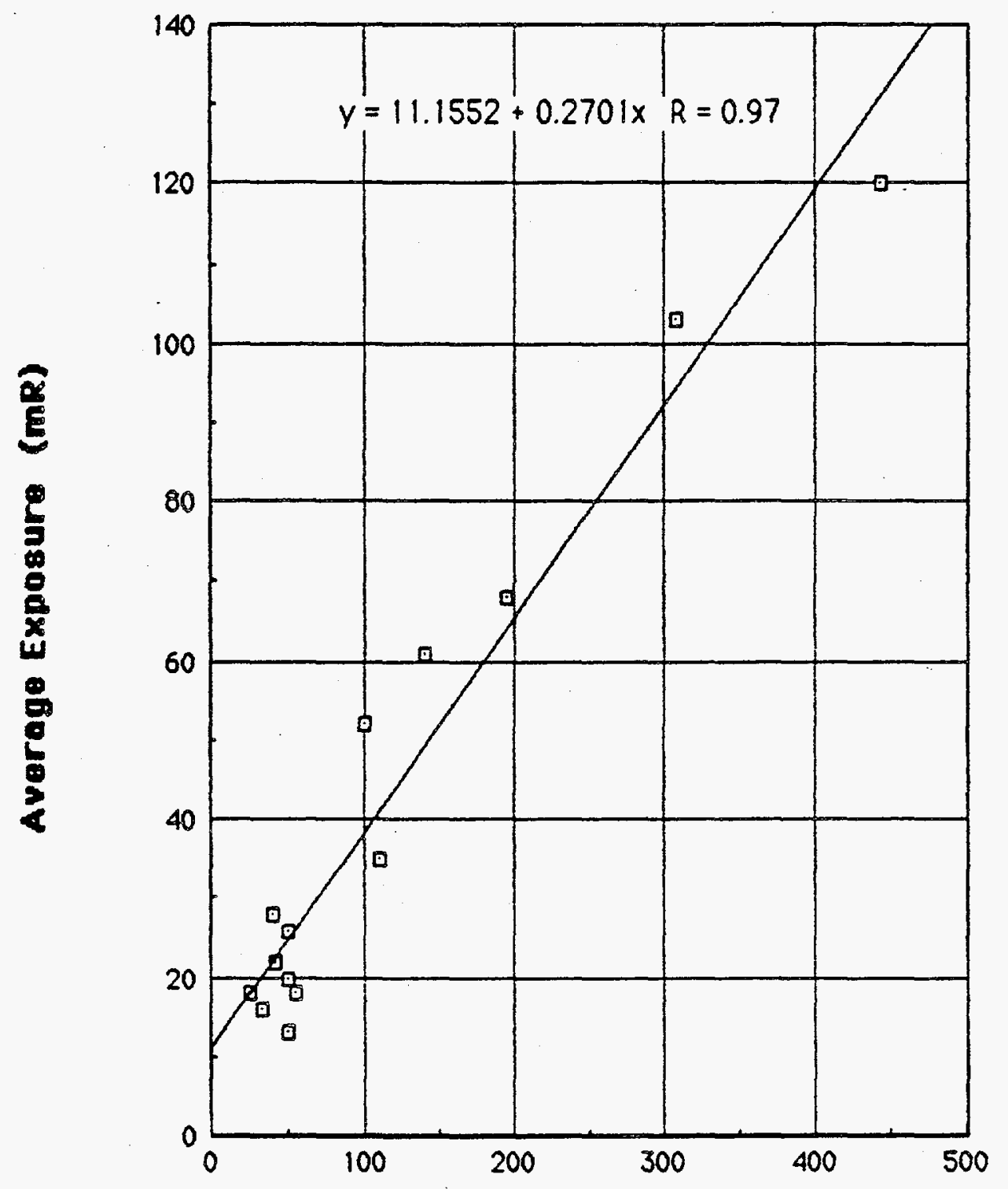

Peak Exposure (mR)

Fig. 4. Average exposure as a function of peak exposure. 


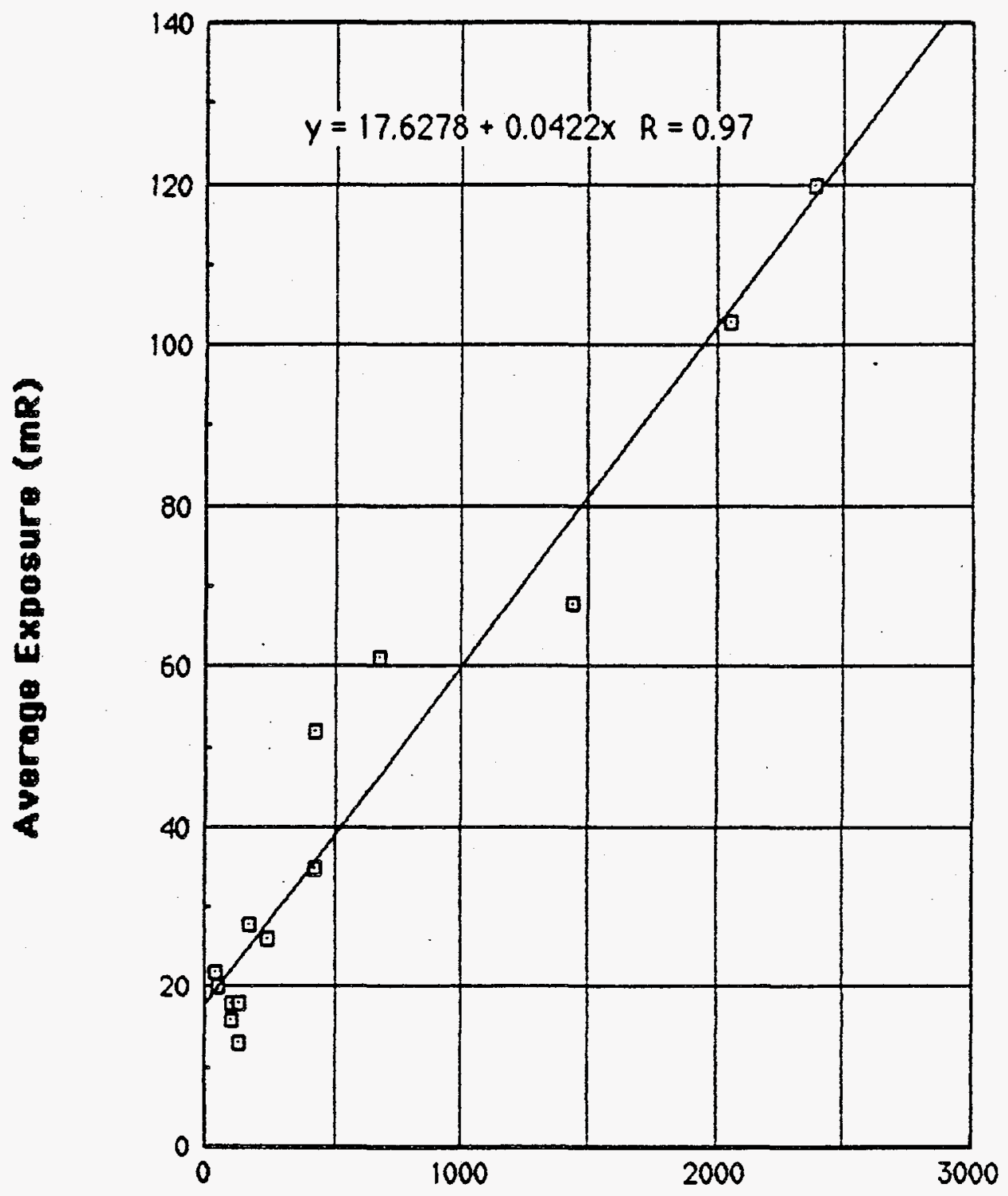

Total Exposure (mR)

Fig. 5. Average exposure as a function of total exposure. 
) 


\title{
APPENDIX D \\ THE DECOMMISSIONING OF THE CERN INTERSECTING. STORAGE RINGS
} (ISR)

\author{
S. Pichler \\ LEP Division \\ CERN \\ Geneva, Switzerland
}

The CERN Intersecting Storage Rings (ISR) was the first facility for colliding proton beams. They were built for a maximum beam momentum of $31.4 \mathrm{GeV} / \mathrm{c}$, giving a center-of-mass energy of $62 \mathrm{GeV}$. The ISR started operation in both rings in 1971 and improved its performance steadily; during high-luminosity runs, operational currents were typically 30 to $40 \mathrm{~A}$, and towards the end of its lifetime it reached 100 times its design luminosity. The total current injected in 1980 and 1981 is shown in Table 1. Ten thousand amperes corresponds to $2 \times 10^{17}$ protons. A review of its performance and of the highlights of its high-energy physics programme has been given by.M. Jacob and K. Johnsen ${ }^{1}$ at the last meeting of the ISR Committee.

TABLE 1. The Total Number of Protons Injected in the ISR in Amperes were:

$\begin{array}{lrc} & 1980 & 1981 \\ \text { RING 1 } & 14,6861 & 9,640 \\ \text { RING 2 } & 12,423 & 11,230\end{array}$

THE INJECTION ENERGY WAS 26 GEV, ACCELERATED TO 31.4 GEV IN BOTH RINGS FOR ABOUT 60\% OF THE OPERATION TIME. THE REMAINING WAS SHARED BETWEEN 26, 22, AND 15 GEV.

In 1981, the CERN Council discussed the long-term scientific.programme of CERN and decided to build the Large Electron-Positron Collider, LEP. In order to accommodate this ambitious project within a constant budget, it was decided in 1983 to stop, among other activities, the ISR and its physics programme. In addition to liberating funds and manpower, this 
also enabled the reuse of certain equipment and especially the fully equipped buildings for LEP, thus alleviating considerably the heavy and urgent demand for hall space for the assembly and storage of LEP machine components and experimental equipment. In order to fulfill this demand, decommissioning of the ISR also meant the dismantling and removal of all machine components and auxiliary equipment from the tunnels and buildings. ${ }^{2}$ These components were partially stored, partially used in LEP and in other CERN projects, and the remainder scrapped.

Of the total of $25,000 \mathrm{~m}^{2}$ of ISR space, $4,000 \mathrm{~m}^{2}$ had to be used for storage, mainly of magnets and vacuum equipment, and $21,000 \mathrm{~m}^{2}$ could be made available for LEP activities.

The decommissioning started in July 1984, and was finished seven months later. During this time, about 1,000 machine components, 2,500 racks and 7,000 shielding blocks had to be moved; about $300 \mathrm{~km}$ of power cables, $3,000 \mathrm{~km}$ of control cables, and $7 \mathrm{~km}$ of stainless steel pipes were dismantled, amounting to a total weight of approximately $30,000 \mathrm{t}$. Elements of machine systems for which no immediate use could be envisaged and which would be essential for a possible reconstruction had to be safely stored. As storage space, the region of the ISR with the beam dump system and the three adjacent octants were chosen: for easy control of the area, good access, and judicious use of the adjacent halls and the remaining tunnel, restricting the handling of highly irradiated components to a minimum.

During the whole decommissioning period, one of the biggest concerns was the handling and disposal of radioactive components; their control and storage was of utmost importance to avoid the dissemination of active material outside radiation-controlled areas or even outside the CERN site. ${ }^{3}$ Therefore, strict rules, which had to be followed rigorously, had to be applied. Fortunately, due to the low loss rates during the 13 years of ISR operation, the maximum integrated dose, measured at $0.3 \mathrm{~m}$ from the vacuum chamber, was only $1 \times 10^{5} \mathrm{~Gy}^{4}$, the radioactivity of the air and water being negligible, and typical dose rates of induced radioactivity, measured 48 hours after a run, were 10 to $50 \mu \mathrm{Sv} \mathrm{h}^{-1}\left(10 \mu \mathrm{Sv} \mathrm{h}^{-1}=1 \mathrm{mrem} / \mathrm{hr}^{-1}\right)$; only in the beam dump and injection areas rates of up to $1000 \mu \mathrm{Sv} \mathrm{h}^{-1}$ were measured. Because of these rather low values, no problems were encountered with respect to doses 
received by the manpower involved in the dismantling. Specific values of radioactivity, measured on samples of different machine components which had to be disposed of, were between 1 and $10 \mathrm{~Bq} \mathrm{~g}^{-1} \mathrm{~F}\left(1 \mathrm{~Bq} \mathrm{~g}^{-1}=27 \mathrm{pCi} \mathrm{gm}^{-1}\right)$, with a maximum of $100 \mathrm{~Bq} \mathrm{~g}^{-1}$ for the heating jackets of the vacuum chamber. In total, about $60 \mathrm{~m}^{3}$ of radioactive waste (stored machine elements, such as main magnets, injection equipment, beam dumps, etc., not included) had to be stored in a controlled area on the CERN site, which is negligible considering the large amount of material dismantled.

For most of the dismantling activities outside labour was employed under the supervision of the CERN staff, and the dismantling of the SFM, a 100 ton analyzing magnet for physics experiments, was entrusted to a salvaging firm. A breakdown is given in Table 2.

TABLE 2. Manpower Requirements and Costs

\begin{tabular}{l|r|r|r}
\hline \multicolumn{1}{c|}{ ACTIVITIES } & \multicolumn{2}{c}{ CERN } & \multicolumn{2}{c}{ CONTRACT LABOR } \\
Man-Hours & Man-Hours & Costs (CHF) \\
\hline Transport and handling & 6,000 & 7,500 & 215,000 \\
Experimental equipment and control cabling & 600 & 12,000 & 330,000 \\
Cooling water pipe work & 2,000 & 10,500 & 256,000 \\
Vacuum equipment and chambers & 2,500 & 8,700 & 207,000 \\
Magnets and cabling & 3,000 & 7,100 & 130,000 \\
Power converters and a.c. cabling & 800 & 4,000 & 115,500 \\
Radiation survey and control & 800 & 780 & 26,000 \\
Administration of salvaging contracts & 100 & 900 & 25,000 \\
Control room and computers & 350 & 360 & 6,800 \\
\hline
\end{tabular}

As already mentioned, the decommissioning of the ISR was done in about seven months. In total, 50,000 man-hours of contract labour and 16,000 man-hours of CERN staff were required for this activity. 
A large part of the most valuable equipment, i.e. auxiliary magnets, power converters, vacuum pumps, will be reused in LEP or in other CERN projects. Equipment which had to be removed and for which no further application could be envisaged for reasons of age or reliability was sold as scrap.

Concerning the financial aspects of the enterprise, it is worthwhile mentioning that the costs for activities contracted outside CERN could be fully financed by the sale of obsolete equipment. The total benefit from equipment and buildings (floor space $21,000 \mathrm{~m}^{2}$ ) can be estimated to be of the order of 57 million Swiss francs.

\section{REFERENCES}

1 M. Jacob and K. Johnsen, "A Review of Accelerator and Particle Physics at the CERN Intersecting Storage Rings," CERN Report 84-13 (1984).

2 S. Pichler, "The Decommissioning of the ISR," Div. Report, CERN LEP-ISR/85-42 (1985).

3 P. Bouriot and A. Perrot (private communication).

4 H. Schönbacher and M. Tavlet, "Radiation Protection Aspects of the Decommissioning of the CERN Intersecting Storage Rings," presented at XIV Regional Congress of IRPA, Dubrovnik, (September 29 - October 2, 1987) and Div. Report TIS-RP/198/CF (10 September 1987). This paper is included as Appendix E.

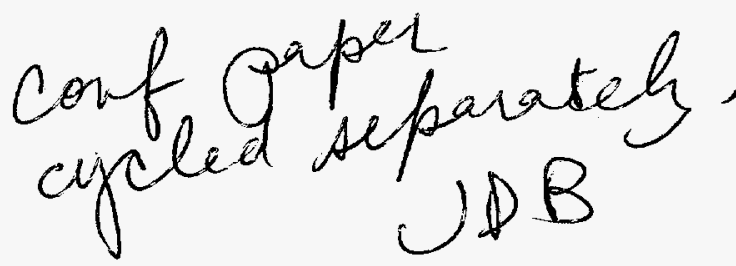


Appendix E removed. Gals

\section{APPENDIX F}

\section{CALCULATIONS ON THE ACTIVATION OF THE CLOSED LOOP COOLING WATER FOR THE COLLIDER DUMPS}

\section{J. D. Cossairt and C. T. Murphy \\ October 2, 1987}

In this note, a calculation of the upper limit for the tritium concentration in the abort dump cooling water system is made.

We assume that the aluminum cylinder is cooled by 180 loops of 0.5 "diameter tubing (see Fig. 3.2 in main text). These loops contain 115 liters of water, which is the volume of water irradiated whenever protons strike the dump.

Using the CASIM output for stars $/ \mathrm{cm}^{3} /$ incident proton produced in various parts of this dump, we find that 20 stars/incident proton would be produced in a cylindrical shell of aluminum of volume 115 liters at the radius of the water loops. Scaling for the different density and cross section for water, there should be $8.6 \mathrm{stars} /$ proton produced in the water.

The activating flux of $2 \times 10^{17}$ protons $/ \mathrm{yr}$ is $6.3 \times 10^{9} \mathrm{protons} / \mathrm{sec}$. The total inelastic cross section is $372 \mathrm{mb}$; the cross section for tritium production is $35 \mathrm{mb}$. Thus the production rate for tritium is

$$
6.3 \times 10^{9} \times 8.6 \times \frac{35}{372}=5.1 \times 10^{9} \mathrm{tritium} / \mathrm{sec}
$$

At equilibrium between production and decay (a condition nearly achieved in 25 years, since the half-life of tritium is 12.2 years), the production rate equals the decay rate. Converting to curies $\left(1 \mathrm{Ci}=3.7 \times 10^{10}\right.$ decays/sec $)$, we find an activation of $0.14 \mathrm{Ci}$ from tritium in the water at equilibrium. There is also an activation of $0.04 \mathrm{Ci}$ of ${ }^{7} \mathrm{Be}$, but this activity decays out quickly.

This activity is diluted into the entire water inventory of the system, which we take to be 115 liters from the cooling coils, 100 liters in the heat exchanger, and 1400 liters in a pair of 2" 
pipes running $350 \mathrm{~m}$ from the dump to the heat exchanger. The specific activity is therefore $84,000 \mathrm{pCi} / \mathrm{ml}$.

The above result is a worst case, assuming that $2 \times 10^{17}$ protons per year are aborted and that the water is not changed in 25 years. If the water is disposed of every two years (typical for Fermilab closed loop systems) the tritium concentration would be a factor of ten less.

We use the methods of Barbier to estimate a dose rate on contact with the tritiated water. Using a "danger parameter" of $10^{-6} \mathrm{mrad} / \mathrm{hr}$, appropriate for the $18 \mathrm{keV}$ electron emitted by tritium which is produced in only $9 \%$ of the stars, we find a dose rate of about $4 \mathrm{mrad} / \mathrm{hr}$. 


\section{APPENDIX G}

\section{COMPOSITION OF SSC DIPOLE COMPONENTS}

Since the composition of materials must be known with some accuracy in predicting activation levels in the SSC environment, those of the dipole coils, collars, and yokes are given below.

1. Inner coil. The $\mathrm{NbTi}$ alloy is $46 \% \mathrm{Nb}$ and $54 \% \mathrm{Ti}$ by weight. The $\mathrm{Cu} / \mathrm{NbTi}$ ratio is 1.3 , again by weight. *

2. Outer coil. The same $46 \% / 54 \% \mathrm{NbTi}$ alloy is used, but the $\mathrm{Cu} / \mathrm{NbTi}$ ratio is 1.8:1.

3. Stainless steel collars. These are of Nitronic 40 Stainless. Other manufacturers sell the same material as 2169 Stainless, with the number indicating the nominal composition $21 \% \mathrm{Cr}, 6 \% \mathrm{Ni}$, and $9 \% \mathrm{Mn}$, with the balance being Fe. A more detailed specication is as follows:

$\begin{array}{ll}\mathrm{C} & 0.08 \% \max \\ \mathrm{P} & 0.06 \% \max \\ \mathrm{S} & 0.03 \% \max \\ \mathrm{Si} & 1 \% \max \\ \mathrm{N} & 0.08 \% \max \\ \mathrm{Mn} & 8 \% \text { to } 10 \% \\ \mathrm{Cr} & 19 \% \text { to } 21.5 \% \\ \mathrm{Ni} & 5.5 \% \text { to } 7.5 \% \\ \mathrm{Fe} & \text { remainder }\end{array}$

4. Steel laminations in yoke. These are made to the ISABEL specifications described in ISA Spec \#94, as follows:

$\begin{array}{ll}\mathrm{C} & 0.008 \% \max \text { after annealing }(0.1 \% \text { before }) \\ \mathrm{P} & 0.03 \% \max \\ \mathrm{Mn} & 0.42 \% \max \\ \mathrm{Si} & 0.05 \% \max \\ \mathrm{S} & 0.05 \% \max \\ \mathrm{Fe} & \text { remainder }\end{array}$

\footnotetext{
* It is common practice in engineering references to quote composition by weight, but without saying that this is the case.
} 